Article

\title{
The Fate of Glutamine in Human Metabolism. The Interplay with Glucose in Proliferating Cells
}

\author{
Jean-Pierre Mazat ${ }^{*}+\uparrow$ (i) and Stéphane Ransac ${ }^{*,+}$ \\ IBGC CNRS UMR 5095 \& Université de Bordeaux, 1, rue Camille Saint-Saëns, 33077 Bordeaux-CEDEX, France \\ * Correspondence: jean-pierre.mazat@u-bordeaux.fr (J.-P.M.); stephane.Ransac@u-bordeaux.fr (S.R.); \\ Tel.: +33-556-999-041 (J.-P.M. \& S.R.) \\ + These authors contributed equally to this work.
}

Received: 22 February 2019; Accepted: 23 April 2019; Published: 26 April 2019

\begin{abstract}
Genome-scale models of metabolism (GEM) are used to study how metabolism varies in different physiological conditions. However, the great number of reactions involved in GEM makes it difficult to understand these variations. In order to have a more understandable tool, we developed a reduced metabolic model of central carbon and nitrogen metabolism, C2M2N with 77 reactions, 54 internal metabolites, and 3 compartments, taking into account the actual stoichiometry of the reactions, including the stoichiometric role of the cofactors and the irreversibility of some reactions. In order to model oxidative phosphorylation (OXPHOS) functioning, the proton gradient through the inner mitochondrial membrane is represented by two pseudometabolites DPH $(\Delta \mathrm{pH})$ and DPSI $(\Delta \psi)$. To illustrate the interest of such a reduced and quantitative model of metabolism in mammalian cells, we used flux balance analysis (FBA) to study all the possible fates of glutamine in metabolism. Our analysis shows that glutamine can supply carbon sources for cell energy production and can be used as carbon and nitrogen sources to synthesize essential metabolites. Finally, we studied the interplay between glucose and glutamine for the formation of cell biomass according to ammonia microenvironment. We then propose a quantitative analysis of the Warburg effect.
\end{abstract}

Keywords: model of central carbon and nitrogen metabolism; flux balance analysis; glutamine; warburg effect; hypoxia

\section{Introduction}

Genome-scale models of metabolism (GEM) greatly help to understand how metabolism varies in different physiological conditions, in different environments, in case of enzyme deficiencies, and in interaction with other metabolisms. Used in conjunction with methods such as flux balance analysis [1], GEM are particularly useful to simulate metabolic changes in large metabolic networks as they do not require kinetic parameters and are computationally inexpensive. Many genome-scale constraint-based models [2-8] have covered central metabolism and have been used successfully to model diseases $[9,10]$. However, the great number of reactions involved in GEM makes it difficult to understand the results obtained in these kinds of studies. In order to have a more manageable tool, we developed a reduced metabolic model of central carbon and nitrogen metabolism, C2M2N, taking into account the true stoichiometry of the reactions, including the stoichiometric role of the cofactors and the irreversibility of some reactions. The configuration used in this work involves three compartments: The extracellular medium, the cytosol, and the mitochondrial matrix. Among 77 reactions, 37 are irreversible and 54 internal metabolites are taken into account. Mitochondrial metabolism (OXPHOS) and mitochondrial transports are often inaccurately represented in GEM with some exceptions [2,11]. In C2M2N, the model of OXPHOS functioning is based on a proton gradient through the inner mitochondrial membrane, represented here by two pseudometabolites DPH $(\Delta \mathrm{pH})$ and DPSI $(\Delta \psi)$. This allowed us to specifically 
take into account the "vectorial" protons [12] across the mitochondrial membrane and not the protons in the reactions inside a given compartment which are, in principle, equilibrated. The main purpose in developing our core model was to create a metabolic model as small as possible, involving the main reactions encountered in experimental metabolic studies to be studied with several theoretical approaches, including the determination of elementary flux modes (EFMs) [13].

To illustrate the practical interest of such a reduced model of metabolism in mammalian cells, we studied glutamine's metabolism and compared it with glucose metabolism. Glutamine is the most abundant amino acid in plasma and has long been recognized to be essential in proliferating cells. Additionally, glutamine has been identified as an alternative to glucose to fuel the Krebs cycle in cancer cells or in hypoxia conditions or mutations [14-20]. The glutamine metabolism goes through glutamate and $\alpha$-ketoglutarate (AKG). Glutamate can be produced in the mitochondria by glutaminase or transaminases, and in the cytosol through nucleotide synthesis or transaminases. When synthesized inside the mitochondria, glutamate exits through the $\mathrm{H}^{+} /$Glutamate cotransporter [21] (the glutamate/aspartate exchanger with a $\mathrm{H}^{+}$entry is sensitive to the $\Delta \mu \mathrm{H}^{+}$and can be considered as irreversible in normal physiological conditions). Inside the mitochondria, glutamine-derived AKG replenishes the tricarboxylic acid (TCA) cycle and can be metabolized either through the canonical forward mode or via reductive carboxylation, leading to citrate and acetyl-CoA in the cytosol. For instance, Chen et al. [22] showed that glutamine enables the survival of mitochondrial DNA mutant cells thanks to both reductive and oxidative pathway in the TCA cycle.

Using flux balance analysis (FBA), we systematically studied and quantitatively discussed all the possible fates of glutamine in central carbon metabolism and demonstrated that glutamine can sustain cell energy production and be used as a carbon and nitrogen source to synthesize essential metabolites, thus contributing to cell proliferation. Here, we show how C2M2N can be used to explore the results of more complex metabolic models by comparing our results with those of MitoCore, a metabolic model of intermediate size built on the same bases.

In a more general approach, we used C2M2N to follow how glucose and glutamine together share energy metabolism and anabolism in different physiological or pathological conditions (proliferating cells, hypoxia). We demonstrated the interest of a core stoichiometric model such as C2M2N in quantitatively tracing the origin of the carbon and nitrogen atoms in the different syntheses and in specifying the respective role of glutamine and glucose in energy metabolism. We demonstrated the role of ammonia release or input or recycling and study some urea cycle dysregulation (UCD). Ultimately, we gave a quantitative account of Warburg effect evidencing the main challenge of proliferating cells, i.e., energy production while maintaining nicotinamide adenine dinucleotide (NAD/NADH) balance.

\section{Short Description of the Metabolic Models}

\subsection{C2M2N Model}

The reactions involved in the metabolic model C2M2N (central carbon metabolic model with nitrogen) are listed in Appendix A and depicted in Figure 1. C2M2N was developed by trial and error from the traditional metabolic networks that can be found in textbooks with the aim to get the smallest network that accounts for most metabolic functions. To this aim, aggregation of consecutive reactions was extensively used when possible while keeping the relevant stoichiometry, particularly of the cofactors. However, C2M2N is not strictly defined, new reactions can be added when necessary (see reaction DH below) or easily suppressed by constraining their flux to zero. The abbreviations of the reaction names are listed in Abbreviations. More precisely, the model consists in (i) a simple version of the Krebs cycle and connected reactions (reactions PDH, CS, IDH2 and 3, SLP, RC2, MDH2, and PYC with the addition of glutamate dehydrogenase (GLUD1)), (ii) in the glycolysis summarized in five steps, G1 (hexokinase + phosphoglucose isomerase), G2 (phosphofructokinase + aldolase + triose-phosphate isomerase), G3 (glyceraldehyde-3P dehydrogenase + phosphoglycerate kinase), ENOMUT (enolase + phosphoglycerate mutase) and PK (pyruvate kinase) extended by the reversible LDH (lactate 
dehydrogenase), and the possible output and input of lactate (LACIO). Gluconeogenesis consists in the reversible reactions of glycolysis plus PEPCK1 (phosphenolpyruvate carboxykinase), GG3 (triose phosphate isomerase + aldolase + fructose-1,6-biphosphatase), and GG4 (phosphogluco isomerase + glucose-6-phosphatase). The mitochondrial phosphenolpyruvate carboxykinase named PEPCK2 was included. The pentose phosphate pathway (PPP) reactions are summarized in PP1 (oxidative part of PPP) and PP2 (nonoxidative part of PPP). The urea cycle is summarized in 4 reactions: CPS1_OTC (carbamoyl phosphate synthase $1+$ Ornithine transcarbamylase), ORNT1 (Ornithine/Citrulline $+\mathrm{H}^{+}$ exchanger), ASS1_ASL_FH (argininosuccinate synthase + argininosuccinate lyase to which we added the fumarate hydratase to convert fumarate in malate as we did for RC2 reaction) and finally ARGASE (arginase). The ornithine synthesis from glutamate and acetylCoA, NAGS_ACY (N-acetylglutamate synthase + Amino Acylase) is completed by the regeneration of acetyl_CoA from acetate by ACS (AcetylCoA Syntethase) and by the ornithine/ $\mathrm{H}^{+}$transporter ORNT2. The urea cycle is supplemented by NOS (NO Synthase).

The synthesis of nucleotide bases (NUC) is represented by a simplification of purine and pyrimidine biosynthesis obtained by averaging the stoichiometry of the different metabolites and cofactors in the metabolic pathway of each nucleotide and taking into account their different amounts in human ( $30 \%$ of $\mathrm{A}$ and $\mathrm{T}$ or $\mathrm{U}$ and $20 \%$ of $\mathrm{G}$ and $\mathrm{C}$ ). It should be stressed that nucleotide synthesis necessitates glutamine which is converted to glutamate and aspartate, itself converted to fumarate and subsequently to malate.

The synthesis of serine from 3-phosphoglycerate involves 3 steps: A dehydrogenase, a transaminase involving the glutamate $/ \alpha$-ketoglutarate pair, and a phosphatase. These three steps are assembled in one reaction: SERSYNT.

The malate-aspartate shuttle (MAS) is fully represented in the direction of NADHc consumption and NADHm production. It involves the malate/ $\alpha$-ketoglutarate exchanger T2 (OGC) and the glutamate/aspartate exchanger T4 (AGC), the malate dehydrogenases (cytosolic MDH1 and mitochondrial MDH2) and the glutamate-oxaloacetate transaminases (cytosolic GOT1 and mitochondrial GOT2). A detailed representation of MAS was mandatory because MAS enzymes are not always operating with the stoichiometry and the direction of MAS for exchange of NADHc for NADHm, i.e., MAS components are not always used to run the MAS as such.

The synthesis of fatty acids is a major pathway in proliferating cells. It starts with citrate lyase (CL) and is represented in the case of palmitate by the reaction PL1 with the corresponding stoichiometries.

The respiratory chain is represented by three reactions: $\mathrm{RC} 1$ which is the respiratory complex I, RC2 (succinate dehydrogenase or complex II which also belongs to the TCA cycle + fumarase), and RC34 which represents complex III + IV.

Finally, the remaining steps of oxidative phosphorylation are represented by ASYNT (adenosine triphosphate (ATP) synthase), ANT, the ADP/ATP exchanger, T5 the Pi carrier, and L the protons membrane leak. The proton gradient is represented by two pseudometabolites DPH $(\Delta \mathrm{pH})$ and DPSI $(\Delta \psi)$. It allowed us to only take into account the "vectorial" protons across the mitochondrial membrane [12]. We introduced a possible $\Delta \mathrm{pH} / \Delta \psi$ exchange (NIG), mimicking the action of nigericin $\left(\mathrm{K}^{+} / \mathrm{H}^{+}\right.$exchanger), or physiologically, the action of ion exchangers in the inner mitochondrial membrane. DPH and DPSI are introduced, when necessary, in mitochondrial carriers equations. ATP hydrolysis is symbolized by ATPASE activity.

Only two entries are considered here, the entry of glutamine (GLNUP) and the entry of glucose (GLUCUP). The possible outputs in this study are pyruvate (T16), serine (SEROUT), aspartate (T14) nucleotides (T13) and palmitate (T17), arginine (T18), urea (T19), and ammonia (T20). The entry of glutamine in the mitochondria goes through the transporter T8. It will give rise to glutamate inside the mitochondria through glutaminase GLS1. 


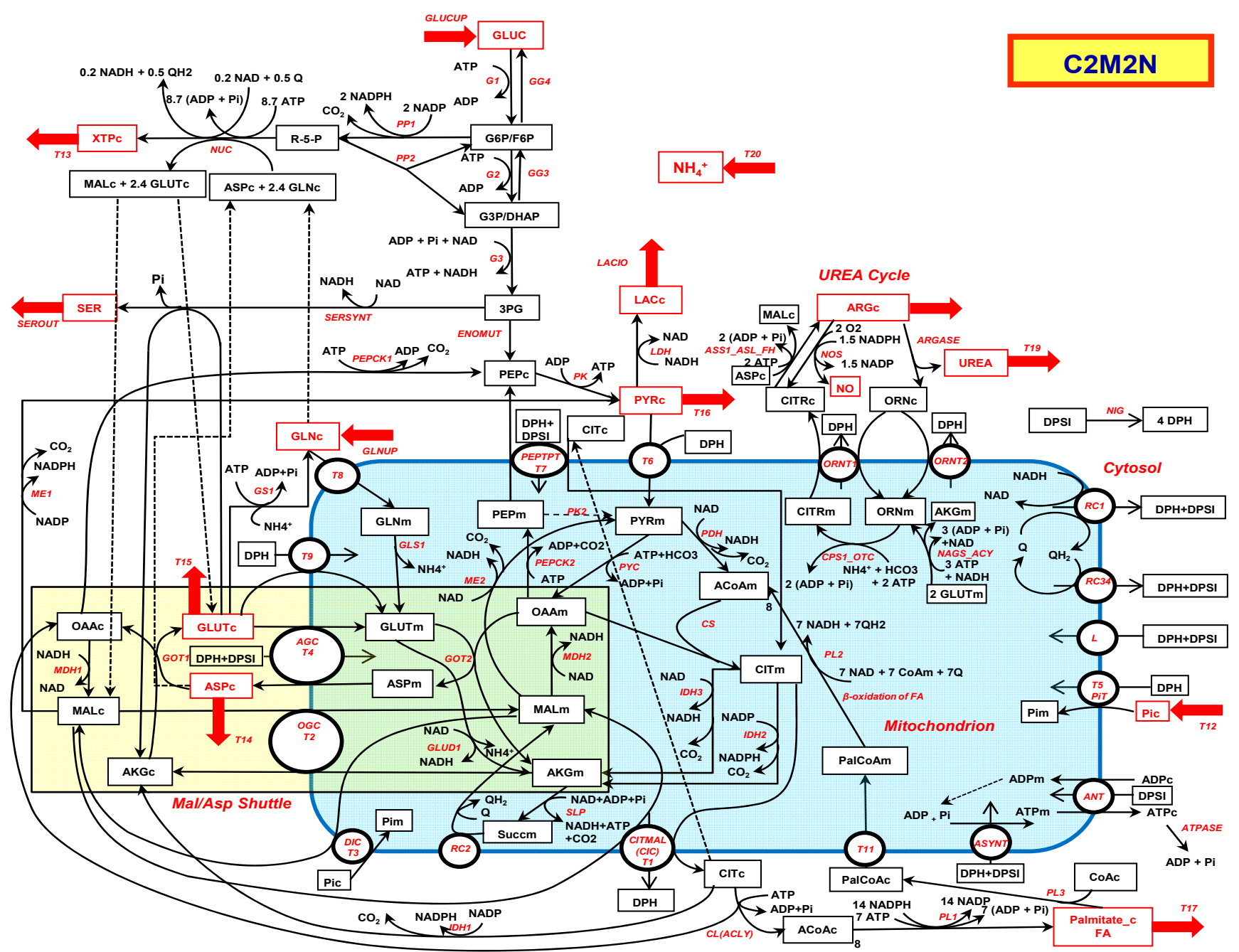

Figure 1. The central carbon metabolic with nitrogen model (C2M2N). Possible entries and outputs are indicated by red arrows. The corresponding metabolites are in red. The abbreviated names of the reactions are indicated in red italic along the arrows of the reactions. The mitochondrion is in blue and the malate-aspartate shuttle in light brown. Dotted lines link identical metabolites duplicated for the sake of presentation. 


\subsection{MitoCore Model}

MitoCore [11] is a manually curated constraint-based computer model of human metabolism that incorporates 324 metabolic reactions, 83 transport steps between mitochondrion and cytosol, and 74 metabolite inputs and outputs through the plasma membrane, to produce a model of manageable scale for an easier interpretation of results. The representation of the proton gradient is nearly the same as in C2M2N with the pseudosubstrates proton motive force (PMF) with DPH $=0.18$ PMF and DPSI $=0.82$ PMF. MitoCore's default parameters simulate normal cardiomyocyte metabolism with entry of different metabolites, particularly glucose and amino acids. These entries were lowered to zero and glutamine or glucose entry were set to one (unless otherwise mentioned) to compare the results of MitoCore with those of C2M2N. (See the SBML file of MitoCore used in this study in the Supplementary Materials).

\subsection{FBA Analysis}

We used FAME (flux analysis and modeling environment) (http://f-a-m-e.org/) [23] to derive the set of fluxes of C2M2N (and of MitoCore for comparison) optimizing the objective functions used below with glutamine or glucose entry for the synthesis of various metabolites. We systematically looked for the absolute flux minimization and then used the flux variability analysis (FVA) to get an idea of the possible other solutions giving the same objective functions. Among these solutions (with a maximal objective function), we selected and drew the one with the maximal ATP synthesis rate. In the main text, the solutions on a simplified version of Figure 1 were represented (Figure 2 and the following). The complete representations of the C2M2N fluxes are in the Supplementary Materials with the corresponding figures labelled Figure Si for the simplified Figure i. The fates of glucose carbons are represented in blue and the fates of glutamine carbons are represented in green. When syntheses arise from both glutamine and glucose other colors are used. Results are given in Table 1.

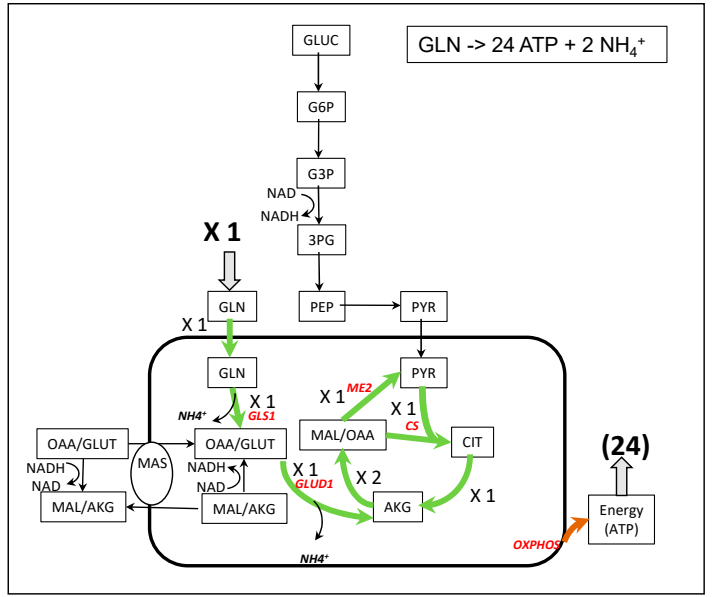

(a)

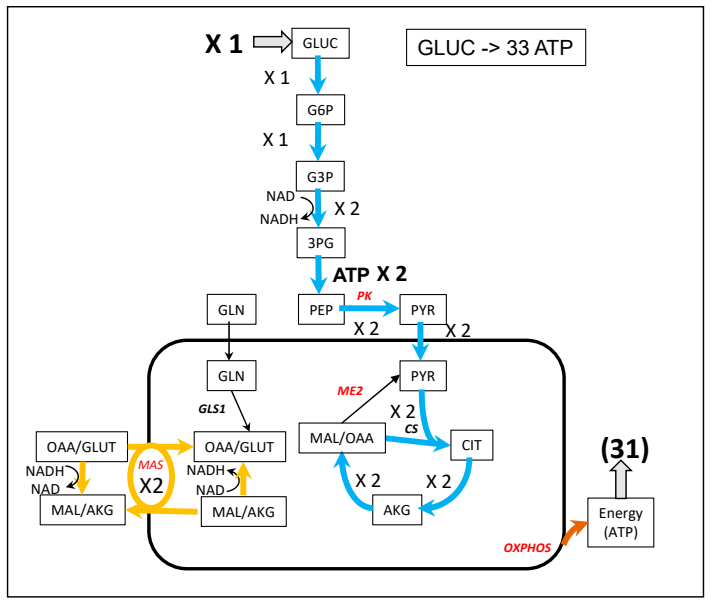

(b)

Figure 2. A simplified representation of ATP synthesis from glutamine (a) and glucose (b). Note that in (b), for the sake of simplicity, the representation of MAS has been separated from the TCA cycle although they share the MDH2 activity with a net flux of 4, 2 for MAS and 2 for the TCA cycle. The complete figures of the fluxes are in Figure S2. 
Table 1. The maximal yield in metabolites synthesis or energy (ATP) from glutamine and glucose at steady state. The first value is obtained with C2M2N and the second with MitoCore.

\begin{tabular}{ccccc}
\hline \multirow{2}{*}{ Objective Function } & \multicolumn{2}{c}{$1 \times$ GLN } & \multicolumn{2}{c}{$1 \times$ GLUC } \\
\cline { 2 - 5 } & Metabolite & ATP & Metabolite & ATP \\
\hline ATPASE & - & $23.8 / 24.0$ & - & $33.34 / 33.04$ \\
Glutamate (T15) & $1 / 1$ & $0 / 0$ & 1 & $8.9 / 8.5$ \\
Pyruvate (T16) & $1 / 1$ & $10.6 / 10.9$ & $2 / 2$ & $6.9 / 6.85$ \\
Aspartate (T14) & $1 / 1$ & $7.6 / 7.7$ & $1.85 / 1.82$ & $2 / 2$ \\
XTP (T13) & $0.6 /-$ & $1 /-$ & $0.86 /-$ & $0 /-$ \\
Palmitate (T17) & $0.17 / 0.17$ & $0 / 0$ & $0.25 / 0.24$ & $0 / 0$ \\
Serine (SEROUT) & $1 / 1$ & $9.3 / 9.1$ & $2 / 2$ & $4.4 / 3.8$ \\
\hline
\end{tabular}

\subsection{Optimization of Proliferating Cells Biomass}

In order to understand how glutamine and glucose share the increase in biomass of proliferating cells, we maximized a biomass function (BM) defined from [24]:

$\mathrm{BM}=0.18$ SERc + 0.11 Palmitate_c + 0.04 XTPc + 0.19 GLNc + 0.17 GLUTc + 0.7 ASPc + 0.05 $\mathrm{ARGc}+0.12 \mathrm{PYRc}+4 \mathrm{ATPc}=>1$ Biomass $+4 \mathrm{ADPc}+4$ Pic.

PYRc takes the place of alanine, ARG summarizes arginine and proline in biomass and also polyamines synthesis, and SER is for serine + glycine.

\section{Results and Discussions}

\subsection{Use of Glutamine for Energy Production (Figure 2)}

Energy production is symbolized by ATPASE activity which is the objective function in this section. About 24 molecules of ATP can be synthesized from 1 molecule of glutamine without any other synthesis (Figure 2a and Figure S2a). One molecule of glutamine enters the mitochondria and then the Krebs cycle as $\alpha$-ketoglutarate (AKG) to double the flux to malate. One molecule of malate generates pyruvate (through ME2) and then acetyl-CoA, which will condense with the OAA derived from the remaining molecule of malate, to generate citrate and a canonical TCA cycle continue in the usual direction with a flux equal to 1 . It is very similar to the glycolysis-derived pyruvate TCA cycle except that, with glutamine, there is no need for NADHc reoxidation. However, ATP synthesis from glutamine necessitates the synthesis of pyruvate either inside or outside mitochondria (see the discussion in $[25,26]$ and is accompanied by a release of ammonia. In the same conditions, one molecule of glucose usually allows the synthesis of 33 ATP molecules at the most (Figure $2 b$ and Figure $\mathrm{S} 2 \mathrm{~b})$. In both cases, the activity of respiratory chain is mandatory in order to reoxidize the NADHm produced by the TCA cycle and to get the maximum rate of ATP synthesis.

\subsection{Pyruvate Synthesis from Glutamine (Figure 3)}

The production of pyruvate (precursor of alanine) from glutamine can follow the production of glutamate and $\alpha$-ketoglutarate (AKG) entering the "left", -oxidative- part of TCA cycle to produce OAA. From OAA, mitochondrial PEPCK2 produces PEP, which exits the mitochondria through citrate and malate cycling (gray arrows on Figure S3a) and generates pyruvate (with PK). One molecule of pyruvate is obtained per molecule of glutamine and 10.6 molecules of ATP can be synthesized in this condition. Another solution without ATP synthesis is depicted in Figure S3c and the more complex solution with MitoCore in Figure S3d. These solutions are analyzed in the Supplementary Materials.

With glucose as substrate (Figure $3 b$ ), the solution is rather simpler, with the synthesis of 2 glycolytic pyruvate and the reoxidation of NADHc by MAS and ETC accompanied by the corresponding ATP synthesis (6.9). 


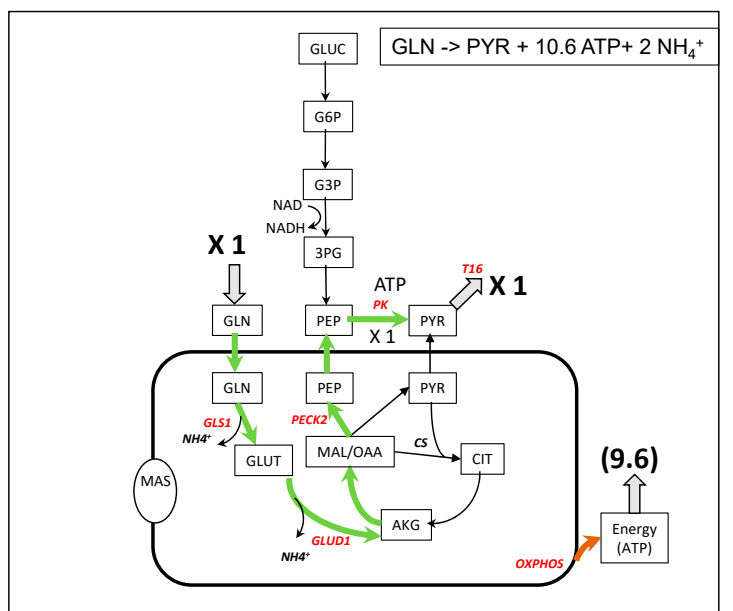

(a)

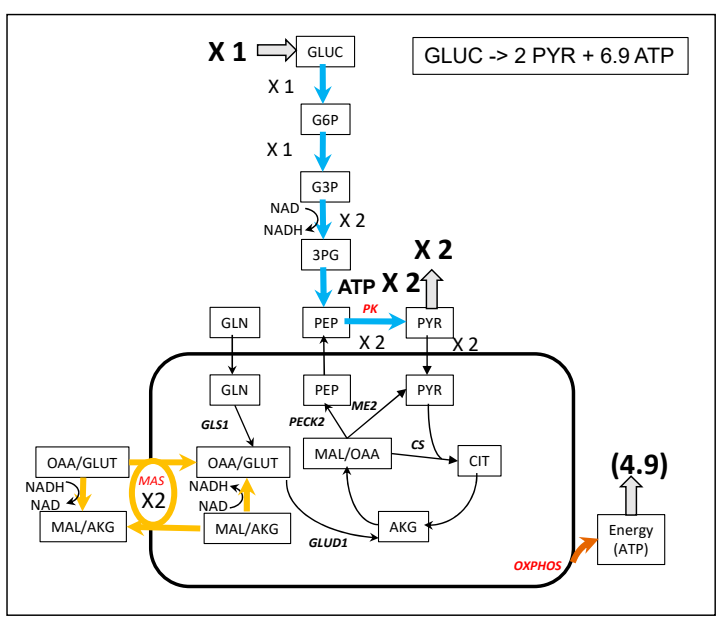

(b)

Figure 3. A simplified representation of pyruvate synthesis from glutamine (a) and glucose (b). Note that in (b), the glycolytic flux and the MAS flux are linked by NAD/NADH cycling. The complete figures of the fluxes are in Figure S3.

\subsection{Apartate Biosynthesis from Glutamine (Figure 4)}

Aspartate is an amino acid which participates in many reactions, particularly in nucleotides synthesis and appears at a high level in the biomass. Due to its low concentration in blood, aspartate synthesis is crucial for cell survival [27-31]. Aspartate synthesis from glutamine (Figure 4a and Figure S4a) involves aspartate amino transferase (GOT) with oxalacetate (OAA) synthesized in the last part of TCA cycle from AKG coming from the glutamine-derived glutamate. Aspartate is carried out of the mitochondria through the glutamate-aspartate carrier (T4). Glutamate is recycled outside the mitochondria by the glutamate/ $\mathrm{H}^{+}$carrier (T9). The maximal yield from one glutamine is one aspartate and 7.6 ATP.

Aspartate synthesis from glucose also requires the activity of GOTs (cytosolic or mitochondrial) but is more complicated (Figure $4 \mathrm{~b}$ and in its full representation in Figure S4b). In the solution of Figure $4 \mathrm{~b}$ and Figure S4b, 1.85 aspartate molecules and 2 glycolytic ATP are synthesized per glucose. Reoxidation of glycolytic NADH imposes the operation of the malate-aspartate shuttle (MAS). NADH cannot be reoxidized by the lactate dehydrogenase because pyruvate carbons are necessary for aspartate synthesis. For this reason, in Figure $4 \mathrm{~b}$ MAS is represented with a flux equal to 2 and the glutamate-aspartate carrier (T4) with a supplementary activity of 1.85 to release the 1.85 aspartate outside the mitochondria. More precisely on Figure S4b one can see that the flux of 2 pyruvate entering mitochondria is split in 1.85 pyruvate carboxylase flux and a 0.15 canonical TCA cycle (thin dark blue arrows in Figure $4 \mathrm{~b}$ and Figure S4b) generating the 1.85 ATPm necessary for the operation of 1.85 pyruvate carboxylase.

In recent papers [27-29], some authors evidenced "an essential role of the mitochondrial electron transport chain ... in aspartate synthesis". This is not unexpected if we consider that mitochondrial synthesis of aspartate requires the synthesis of OAAm (using pyruvate carboxylase with ATP or malate dehydrogenase generating $\mathrm{NADH}$ ) and the fact that the GLU/ASP exchanger (T4) depends on the $\Delta \mu \mathrm{H}^{+}$, i.e., the ETC activity. We can confirm this result in our model as represented in Figure $4 \mathrm{c}, \mathrm{d}$, which depicts the synthesis of aspartate as a function of ETC activity with one glutamine or one glucose. In both cases, the flux of aspartate synthesis proportionally decreases with the decrease of the activity of respiratory chain (modulated by RC34 activity). As shown experimentally, this is due in part to the necessity to reoxidize NADHc. Introducing in C2M2N a dehydrogenase activity (DH) reoxidizing NADHc, we induce an increase of aspartate synthesis in accordance with the results in [29].

Other reactions also appear essential such as the pyruvate carboxylase (PYC) and the pentose phosphate pathway. 


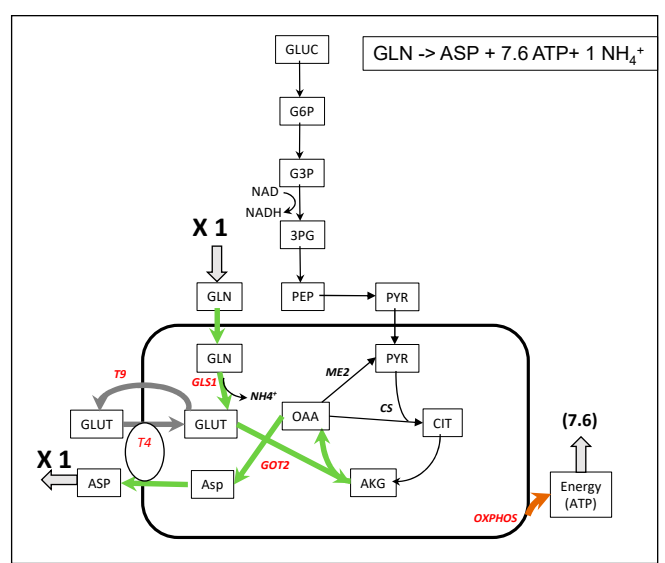

(a)

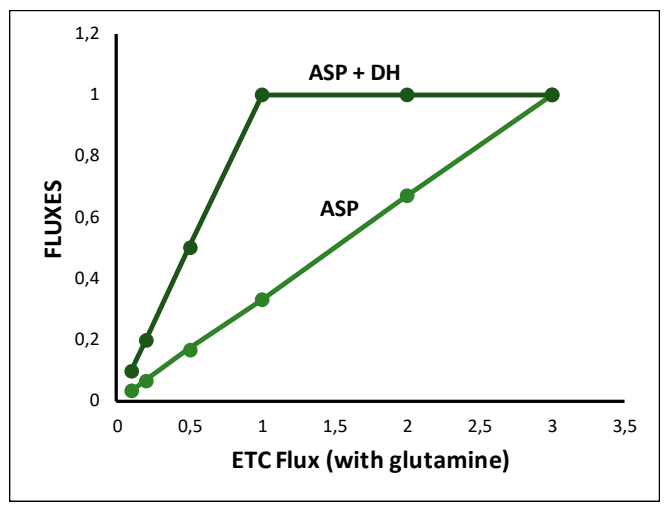

(c)

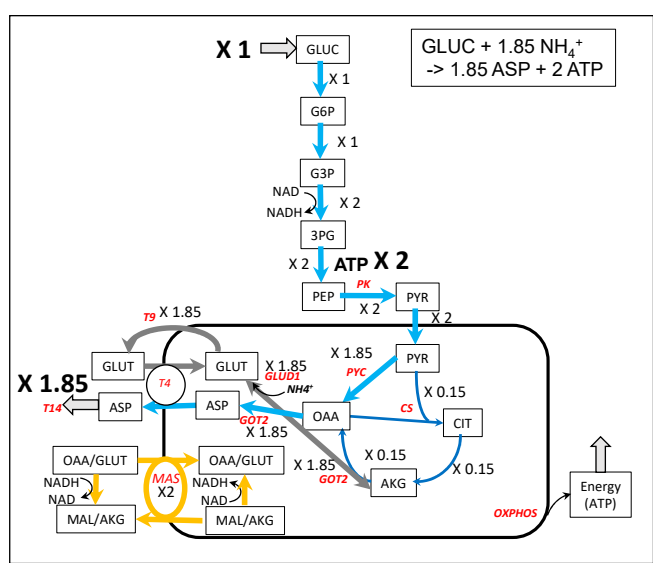

(b)

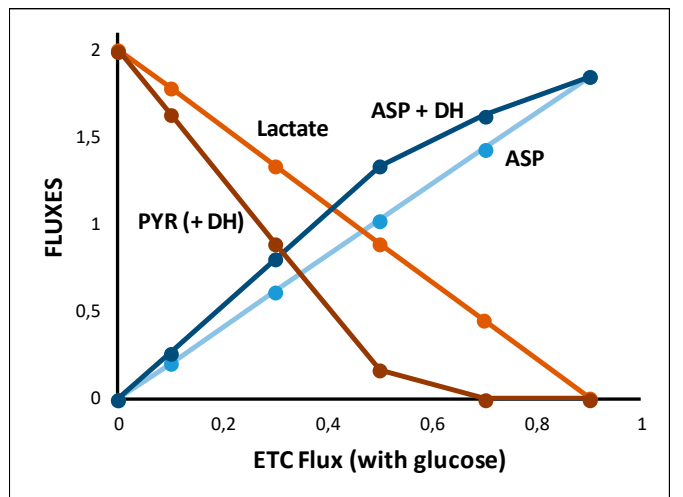

(d)

Figure 4. A simplified representation of aspartate synthesis from glutamine (a) and glucose (b). Note that in (b), the flux through T4 and GOT 2 can be split in a malate-aspartate shuttle (MAS) with a flux of 2 and in a 1.85 flux through T4 corresponding to aspartate output, i.e., T4 net flux is 3.85. See the complete representation of the glutamine- and glucose-derived aspartate in Figure S4a,b. (c): ETC inhibition (RC34 is inhibited) of aspartate synthesis from glutamine in the absence or presence of the dehydrogenase DH which reoxidizes NADHc. (d) ETC inhibition (RC34 is inhibited) of aspartate synthesis from glucose in the absence or presence of the dehydrogenase $\mathrm{DH}$ which reoxidizes NADHc. The ETC inhibition leads to lactate release, or pyruvate release in the presence of $\mathrm{DH}$.

\subsection{Nucleotide Synthesis from Glutamine and Glucose (Figure 5)}

The synthesis of nucleotides (XTP) requires glutamine, aspartate, and R5P. In the absence of ASP and R5P, glutamine has to be used to ensure these syntheses. In the solution of Figure 5a, 0.6 XTP is synthesized that necessitates 1.44 glutamine. To do so, the input of one glutamine is used with, in addition, a 0.44 cycling of glutamate/glutamine via glutamine synthase (GS1). The glutamate resulting from glutamine entry (flux $=1$ ) is entirely transformed in PEP then R5P through gluconeogenesis and pentose phosphate pathway. This solution necessitates a slight entry of $\mathrm{NH}_{4}{ }^{+}(0.04)$ and is accompanied by the production of 0.98 ATP.

The synthesis of nucleotides from glucose requires R5P synthesis and the recycling of glutamine and aspartate. R5P is produced by the pentose phosphate pathway. Glutamine is recycled from glutamate $\left(+\mathrm{NH}_{4}{ }^{+}\right)$by glutamine synthase (GS1) and aspartate is recycled from fumarate then malate and oxaloacetate. There is the need to reoxidize glycolytic NADHc and the NADHc produced by nucleotide synthesis inducing a 0.74 flux through the malate-aspartate shuttle (see Figure $5 \mathrm{~b}$ and Figure S5b). The yield of nucleotide synthesis with glucose is higher than with glutamine (0.86 XTP per glucose) but with a high input of $\mathrm{NH}_{4}{ }^{+}$and no ATP production despite a higher activity of respiratory chain. 


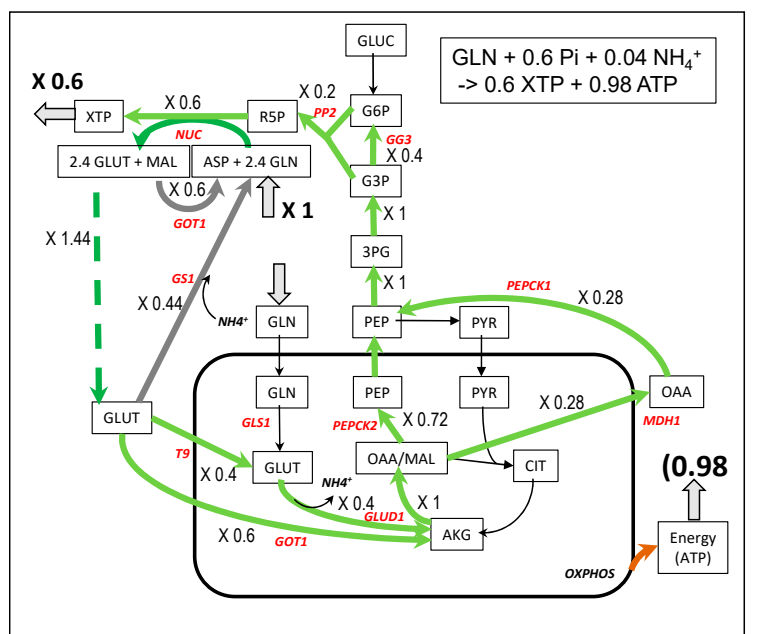

(a)

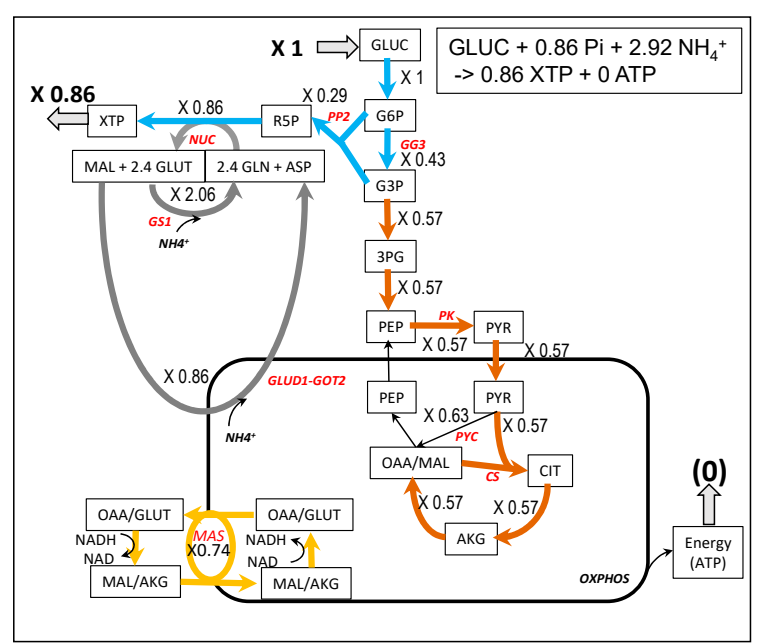

(b)

Figure 5. A simplified representation of nucleotides synthesis from glutamine (a) and glucose (b). Note that in (b), the flux through T4 and GOT 2 can be split, in the malate-aspartate shuttle (MAS) with a flux of 0.74 and in a 0.86 flux corresponding to aspartate recycling. The complete model is in Figure $\mathrm{S} 5 \mathrm{a}, \mathrm{b}$ in the Supplementary Materials.

\subsection{Fatty Acids Synthesis from Glutamine and Glucose (Figure 6)}

Fatty acids (FA) synthesis is an important pathway in proliferating cells, especially for phospholipids syntheses. In the case of palmitate synthesis, 0.17 palmitate molecule can be synthesized from one molecule of glutamine and because this is rather energy consuming, no ATP molecule is synthesized (Figure 6a). The synthesis occurs through ATP citrate lyase (CL) fed by citrate synthesized in TCA cycle. The glutamine-derived AKG flux in the TCA cycle (flux $=1)$ is split between the reductive pathway giving directly citrate by inversion of IDH3 (flux $=-0.4$ ) and the oxidative pathway through malate and oxalacetate (flux $=0.6$ ) and pyruvate coming from the recycling of OAAc, product of CL in the cytosol. To these fluxes is superimposed a cycle involving IDH1 and IDH3 in order to transform NADHm into NADPHc necessary for the synthesis of fatty acids (flux $=2.4$ in gray in Figure 6a and Figure S6a) giving a net reductive flux of IDH3 equal to -2.8. NADPHc could have been made by the malic enzyme (ME1) in the cytosol but this occurs with a slightly lower yield (0.16 PAL per 1 glutamine). In [32], the authors showed that $60 \%$ of NADPH is synthesized from glutamine by malic enzyme and that the pyruvate produced is excreted as lactate. We do not observe a release of lactate in our models because, in the absence of glucose, there is no excess of carbon and pyruvate enters the mitochondria to replenish the TCA cycle in the classical anaplerotic way. The authors also observed a G6PDH flux (PP1) of the same order as the glutaminolysis flux, demonstrating that several sources of NADPH can be operating in vivo at the same time.

The involvement of reductive use of glutamine and participation of IDH1 in fatty acids synthesis is well recognized $[14,15,33]$ in hypoxia and with mutations in TCA cycle or in respiratory chain [34], and appears to be linked to the AKG/citrate ratio [35]. In our model (Figure 6a), the TCA cycle can be viewed as converging to citrate synthesis by the two oxidative and reductive pathways from AKG. This dual TCA pathway used for citrate synthesis is also well documented [36,37]. 
With glucose as carbon substrate (Figure 6b), oxalacetate coming from ATPcitrate lyase (CL) generates malate thanks to the cytosolic malate dehydrogenase (MDH1), oxidizing the glycolytic NADHc as found in $[34,38]$. The cytosolic malate re-enters mitochondria through antiporters, particularly in exchange with citrate. It is an elegant way to absorb the cytosolic reductive power of glucose avoiding the operation of MAS. This pathway is often observed when NADH reoxidation is impeded (respiratory complex deficiency for instance). Like with glutamine, there is a cycle involving IDH1 and IDH3 which transforms NADHm into NADPHc (flux $=3.5$ in gray in Figure $6 \mathrm{~b}$ and Figure S6b).

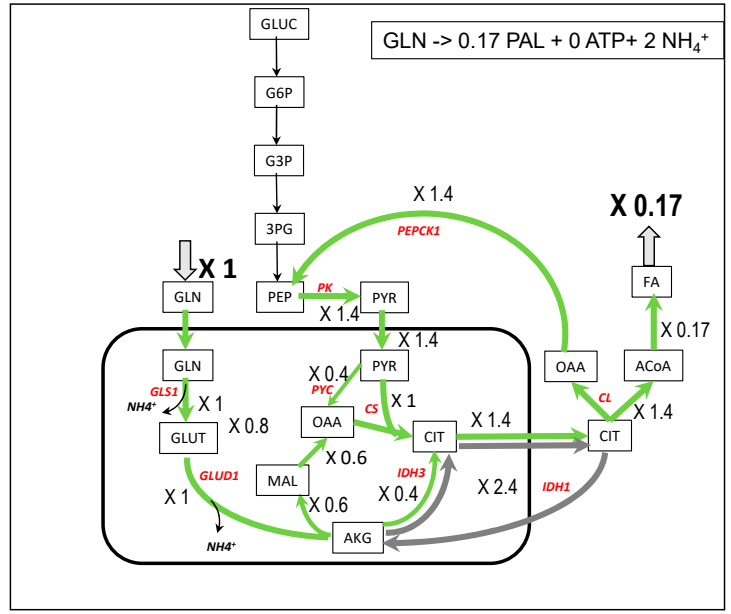

(a)

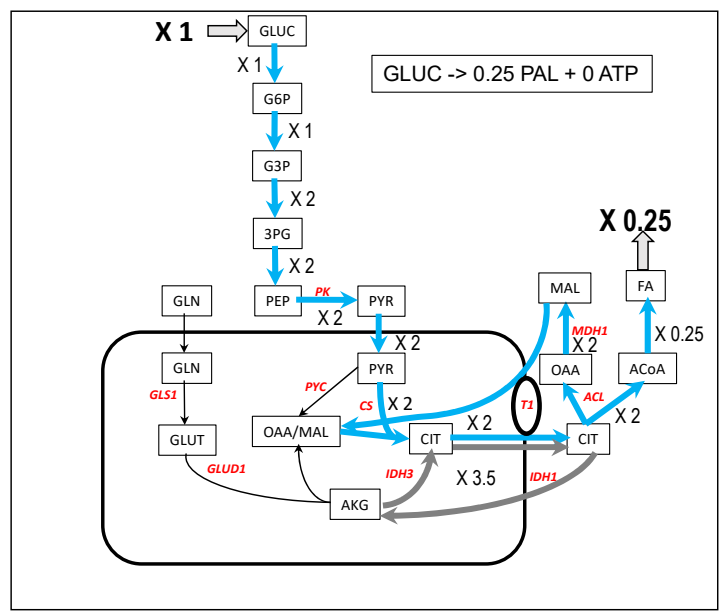

(b)

Figure 6. A simplified representation of palmitate synthesis from glutamine (a) and glucose (b). In gray, cycling of citrate accompanying the conversion of NADHm in NADPHc needed for palmitate synthesis. The complete model is in Figure S6a,b in the Supplementary Materials.

\subsection{Serine Synthesis from Glutamine and Glucose (Figure 7)}

Serine is the major source of one-carbon units for methylation reactions via tetrahydrofolate and homocysteine. It is the precursor of glycine (see [39] for a mini-review). In his pioneering work, Snell demonstrated the importance of serine biosynthesis in rat carcinoma [40]. More recently several authors emphasized the role of serine in breast cancers and in melanoma cells [41-43].

We have already studied the different yield in serine synthesis on different substrates with our core model [44]. The synthesis of serine from glutamine is represented in Figure 7a. It involves the synthesis of PEP as in pyruvate synthesis, but here PEP is used to make 3PG, a serine precursor. The glutamate used in the transamination reaction is recycled from the AKG produced and the NADH produced by the 3-phosphoglycerate dehydrogenase reaction is reoxidized with MDH1. In addition, 9.3 ATP can be synthesized. With glucose as a carbon substrate, 3PG is synthesized directly from glucose via glycolysis (Figure 7b). However, the work of Hanson in rats demonstrates that "pyruvate entry into the gluconeogeneic pathway is the major route for serine biosynthesis" [39,45]. It is difficult to obtain this pathway from glucose in C2M2N. This could indicate that in the case of this work, serine is mainly synthesized from other sources than glucose (glutamine or other amino acids, for instance). The yield of serine synthesis with glucose as a carbon substrate can reach 2 molecules of serine per molecule of glucose with 4.4 ATP molecules produced. 4 NADHc are generated which requires a flux of 4 in the MAS (Figure 7b). 


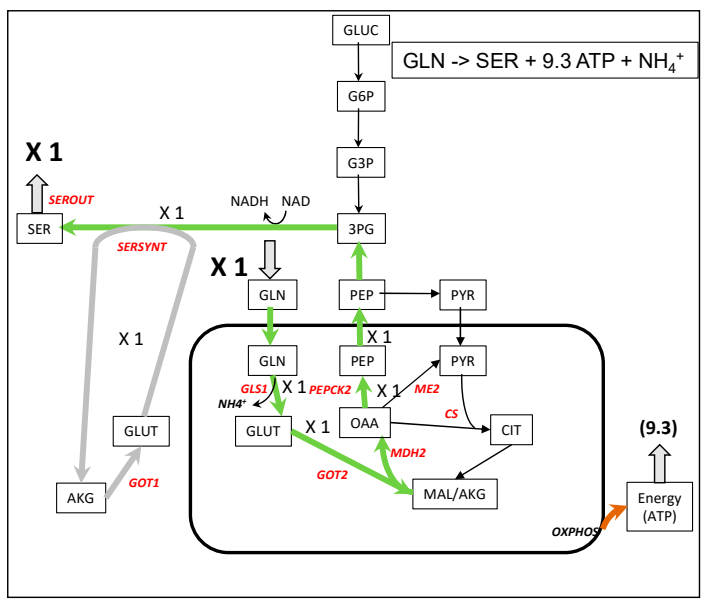

(a)

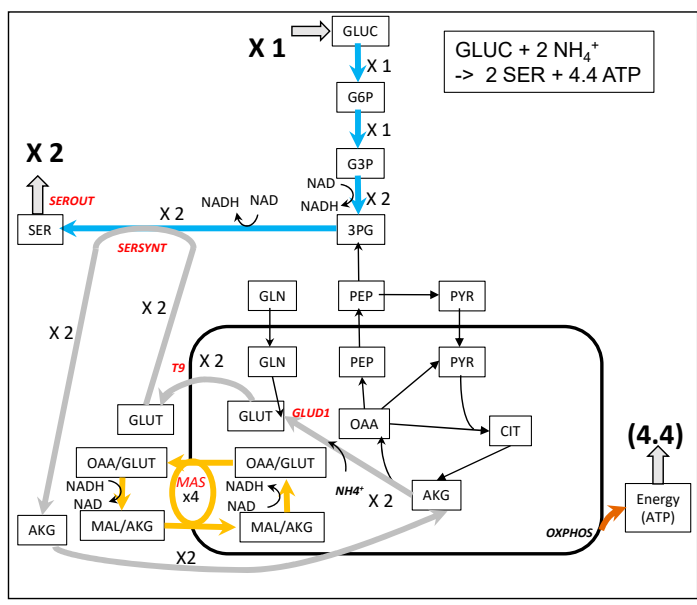

(b)

Figure 7. A simplified representation of serine synthesis from glutamine (a) and glucose (b). In light gray, is the recycling of glutamate for serine synthesis. The complete model is in Figure S7 in the Supplementary Materials.

\subsection{Comparison of C2M2N with MitoCore}

Similar FBA simulations have been performed with MitoCore which is a more detailed model of metabolism. The results are nearly identical and are presented in Table 1 . Note that the synthesis of nucleotides is not included in MitoCore, so it is not possible to compare XTP synthesis in both models. The SBML file of the version of MitoCore used in this paper (with only input of glutamine or glucose) can be found in the Supplementary Materials.

\subsection{Distribution between Glutamine and Glucose for the Biomass of Proliferating Cell: The Nitrogen Metabolism}

Proliferating cells (cancer or immune cells) necessitate the input of $\mathrm{NH}_{3}$ group for the synthesis of amino acids, nucleic acids, and polyamines. It is widely accepted that glutamine is the main donor of nitrogen for proliferating cell with a central role of glutamate. We have shown above that glutamine as well as glucose can allow the synthesis of most of the essential metabolites which constitute the biomass of proliferating cells and supply cell energy (ATP) [26]. In the tumor and immune cells microenvironment, glutamine and glucose are simultaneously present and the question arises about how they divide the task of feeding biomass. To answer this question, we used the biomass as the specific objective function of proliferating cells. We let free the uptake of glucose and glutamine, only dictated by the optimization procedure of the biomass, but with an arbitrary limit of 1 , to avoid unlimited amount of biomass. We obtained a metabolic pattern (Figure 8a and Figure S8a) in which the cell incorporated a large amount of glutamine not only for $\mathrm{NH}_{4}{ }^{+}$feeding, but also in part for carbon supply and around $80 \%$ of ATP to sustain biomass formation as noticed in [26] (See the ATPc and NADHm balance in Figure S8a). This occurs with a large output of ammonia close to the input of glutamine which is larger than the need in $\mathrm{NH}_{3}$ for the biomass as already noted [14,32,46]. Although ammonia is toxic for the cell, it has been demonstrated that cancer cells accumulate ammonia in their microenvironment at rather high concentration [47,48]. In [47], the authors show that, actually, cancer cells released ammonia when ammonia concentration in their microenvironment is low and uptook ammonia for higher ammonia concentrations with a threshold of around $1 \mathrm{mM}$. Figure $8 \mathrm{a}$ corresponds to low ammonia concentration in the microenvironment. An easy way to simulate the high concentration in the microenvironment associated with an ammonia input is to prevent ammonia output from the cells. In these conditions, the uptake of glucose and glutamine to generate one unit of biomass are similar (0.762 and 0.883 ) (Figure $8 \mathrm{~b}$ and Figure S8b). In this simulation $100 \%$ of ATP derives from glucose as calculated from the origin (glucose or glutamine) of NADHm (see Figure 
S8b). AKGm derives entirely from glutamate by transamination (GOT2). The main salient point of this solution is the recycling of $\mathrm{NH}_{4}{ }^{+}$released by glutaminase and incorporated in part of the AKGm, mainly by the reversion of glutamate dehydrogenase, as shown experimentally by Spinelli et al. (see Figure $1 \mathrm{~F}$ in [47]), and also to a lower extent by carbamyl-phosphate synthesis (CPS1_OTC). No output of $\mathrm{NH}_{4}{ }^{+}$is observed. Another advantage of $\mathrm{NH}_{4}{ }^{+}$incorporation by glutamate dehydrogenase is the reoxidation of part of NADHm so that the glutamine per se does not contribute to the mitochondrial redox load (see the NADHm balance due to glucose or glutamine in Figure S8b).

Glutamine limitation suppresses cancer cell proliferation which can be overcome by supplementing cells with ammonia [46,47]. It is easy to simulate this situation by suppressing the entry of glutamine inside the cells (Figure 8c and Figure S8c). In these conditions the optimization of the biomass objective function relies on a massive entry of ammonia and glucose which is now the sole source of carbon and energy. As proposed in [49], this situation can occur when ammonia released locally by the catabolism of glutamine diffuses in the microenvironment to cells, for which the glutamine concentration in the microenvironment is lower.
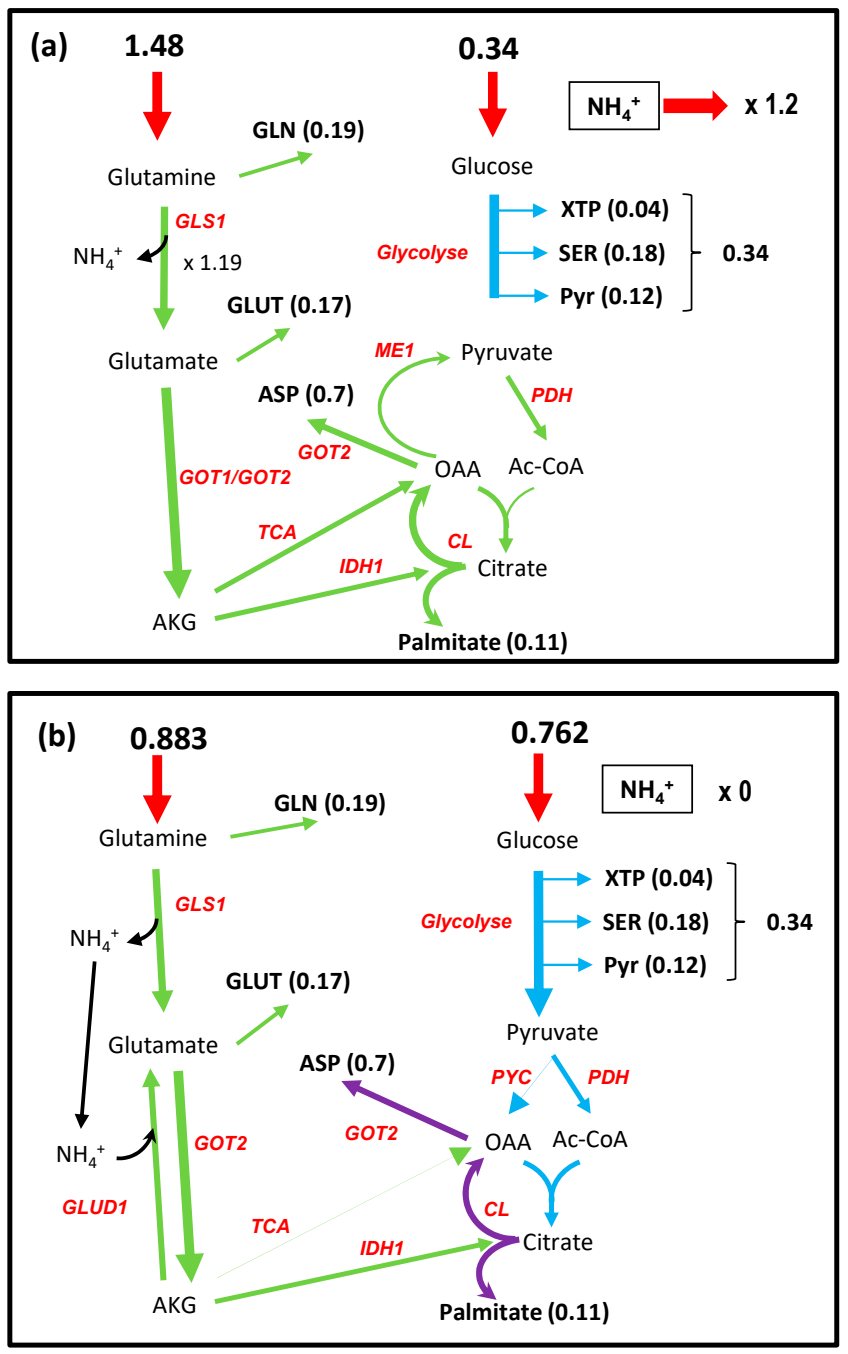

Figure 8. Cont. 


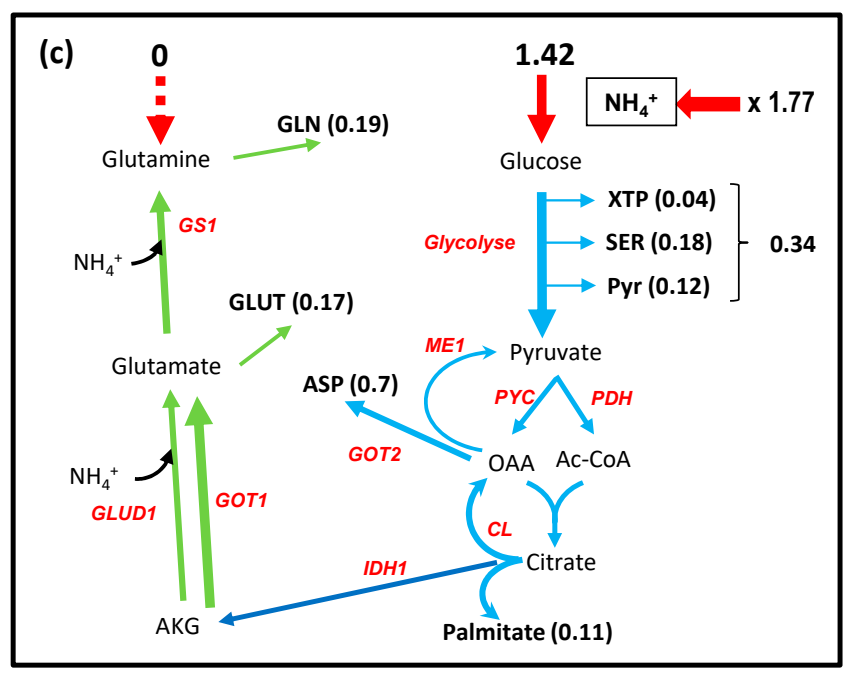

Figure 8. The interplay between glutamine, ammonia, and glucose to sustain cell proliferation. (a) Nitrogen is incorporated as a large excess of glutamine with release of ammonia. (b) Ammonia produced by GLS1 is stoichiometrically recycled by GLUD1(glutamate dehydrogenase) in the reverse direction. In (c), nitrogen is incorporated as ammonia in glutamate by the reversion of glutamate dehydrogenase and in glutamine by glutamine synthase. A small part of $\mathrm{NH}_{4}{ }^{+}$is incorporated directly by CPS1 (carbamyl phosphate synthase). The complete metabolic networks are in Figure S8a-c.

If, using FVA, we look at essential reactions (those which are strictly positive or strictly negative and whose inhibition is susceptible to inhibit biomass formation) we obtain the reactions allowing biomass synthesis, i.e., CL, NUC, PL1, SERSYNT, ASS1_ASL_FH, and T12 which represent more reactions due to the fact that most of these reactions stand for several consecutive reactions. All of which are therapeutically targeted in cancer [50]. Curiously, GLS1 and GLUD1, the first steps in glutaminolysis, do not pertain to this set of reactions, indicating that other solutions which do not use these reactions exist.

If we put GLS1 to zero we obtain a solution in which $\mathrm{NH}_{4}{ }^{+}$is incorporated from the microenvironment instead of glutamine by the reversion of Glud1 (similarly to Figure $8 \mathrm{c}$ and Figure S8c). If, in addition to GLS1 inhibition, we add the inhibition of Glud1, we still have the normal biomass production of 1 with all glutamine metabolized in glutamate by the nucleotide synthesis NUC with excretion of nucleotides. In these conditions, it could be therapeutically pertinent to inhibit, simultaneously, nucleotide synthesis and glutamate dehydrogenase Glud1.

\subsection{Rewiring Urea Cycle Metabolism.}

Urea cycle is another way to get rid of ammonia and to insure particular syntheses. The complete urea cycle takes place in the liver. However, some urea cycle enzymes are expressed in different tissues, particularly in proliferating cells, in order to provide cells with arginine, ornithine, citrulline, and polyamines. Overexpression of arginase 1 has been described in different types of tumors [51,52]. As pointed out in [53], by deprivation, "arginine becomes an essential amino acid generating a vulnerability that is utilized to treat cancer using arginine-deprivation agents".

We can show (Figure 9) that increasing the arginase activity while limiting the input of glutamine at the initial level induces a decrease in the biomass by diminishing the availability of arginine. However, glutamine can overcome arginine deficiency by a net arginine synthesis from glutamate conferring resistance to arginine deprivation with "higher glutamate dehydrogenase and glutaminase expression and preferential vulnerability to glutamine inhibitors" [54]. 


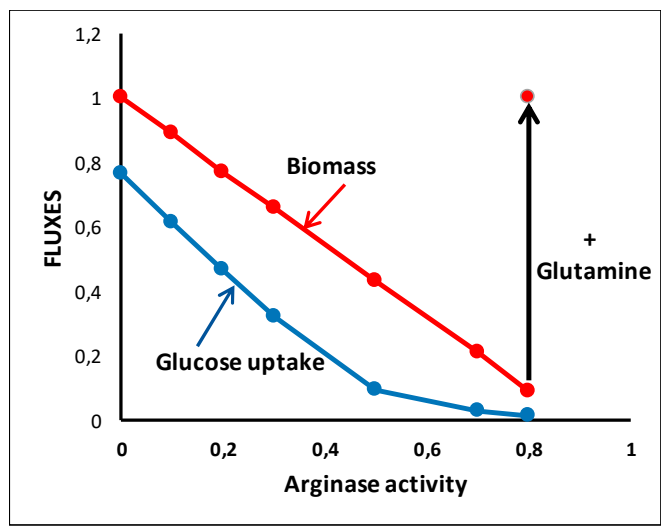

Figure 9. Arginine deprivation. Arginine deprivation is obtained with increased expression of Arginase in the situation of Figure $8 \mathrm{~b}$ with the same input of glutamine (0.883). An increase in glutamine input (1.683) restores the biomass to 1.

\subsection{Quantitative Aspects of the Warburg Eeffect}

The Warburg effect or aerobic glycolysis is a salient feature of most cancer cells. It is characterized by an increase in glucose uptake and lactate release in the presence of oxygen. To simulate an increase in cell proliferation, we started from the situation of Figure 8a, i.e., a maximum of biomass equal to 1 with free availability of glucose and glutamine and we supposed that this situation corresponds to the maximum capacity of the respiratory chain (ETC flux $=2.374$ ). Then, we simulated an increase of biomass. The solution obtained is represented in Figure 10a. When biomass increased from 1, a massive glucose input was observed that supplements the extra need for ATP. It was accompanied by a massive release of lactate to reoxidize glycolytic NADH, a task that can no longer be provided by the respiratory chain. We also observed an increase of glutamine uptake (accompanied by an increase in $\mathrm{NH}_{4}{ }^{+}$release) necessary for the biomass increase because the additional glucose carbons were lost with lactate excretion.

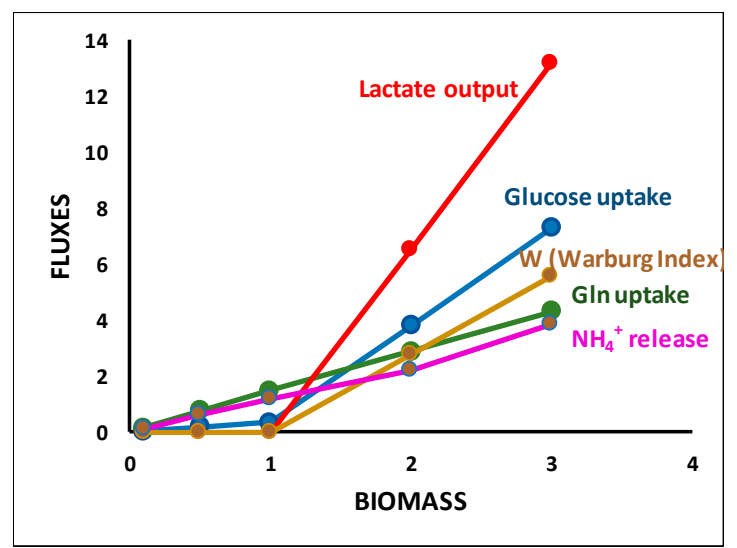

(a)

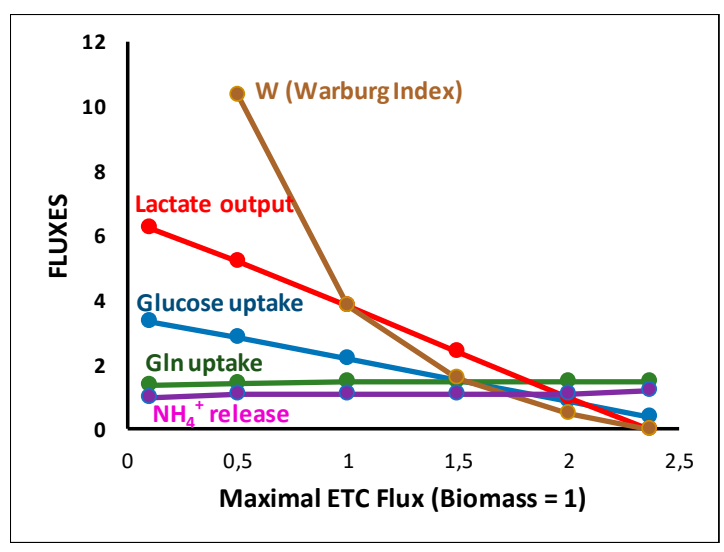

(b)

Figure 10. The Warburg effect. (a) Increase in biomass at constant respiratory chain content (ensuring maximum biomass equal to 1 ). (b) Decrease in respiratory rate from the sufficient value (2.374) to insure ATP synthesis and NADHc reoxidation. W is an index measuring the Warburg effect defined in [55] as the ratio of lactate release over respiratory chain (RC34) activity.

We can take this problem the opposite way (Figure 10b), still asking for a biomass equal to one but inhibiting the respiratory rate from its normal value, 2.374 , which simulates hypoxia. As in Figure 10a, an increase in glucose uptake was observed that compensates the decrease in respiratory rate accompanied by a greater increase in lactate release but a slight decrease in glutamine. 
As noticed in $[29,55]$, the Warburg effect appears as a respiratory chain inadequacy (not necessary a decrease) in proliferating conditions leading to an increase of glycolytic flux to provide the requisite extra amount of ATP and, consequently, an increase in lactate output to reoxidize the extra amount of NADHc. Dai et al. [55] also emphasize the "redox balancing in leading to the Warburg effect and its correlation to proliferation rate".

\section{Conclusions}

We developed a core metabolic model of central carbon and nitrogen metabolism, C2M2N, with a limited number of reactions (77) and of metabolites (54 internal metabolites) to explore the potential of glutamine to supplant glucose in metabolic syntheses, energy and biomass production. A salient feature of this model is that it takes into account the actual stoichiometries of the reactions particularly the stoichiometries of cofactors, even for concatenated reactions. A second characteristic of this model is to consider mitochondria as an isolated compartment with the relevant transporters. This led us to the third characteristic, to develop a relevant model of oxidative phosphorylation taking into account the mitochondrial $\Delta \mu \mathrm{H}^{+}$in the form of pseudosubstrates DPH and DPSI as was already done in MitoCore [11] and in [2]. The last characteristic of C2M2N is that it takes into account nitrogen metabolism in addition to carbon metabolism. This led us to an easily tractable model that can produce rigorous quantitative simulations and testable predictions taking into account the constraints due to the regeneration of cofactors and a compromise between metabolite synthesis and energy production. Note that the model is not fixed; reactions can be added or cancelled to answer specific questions, for instance, introduction of $\mathrm{DH}$ reaction to simulate dehydrogenase activities that are able to regenerate NADc [29] in the synthesis of aspartate (Figure 4c,d).

The advantage of $\mathrm{C} 2 \mathrm{M} 2 \mathrm{~N}$ is that, due to the low number of reactions and metabolites, the interpretation of the results is rather straightforward and can be easily represented and understood on a metabolic scheme. All the solutions maximizing an objective function (described by FVA) can be more easily explored, as done in the Supplementary Materials, in the case of pyruvate synthesis, and in Section 3.8 in the case of biomass formation. Furthermore, quantitative balance of any internal metabolites can be performed as in Section 3.8 for biomass production. Furthermore, when glutamine and glucose are used together, it is possible to distinguish which one contributes to any particular synthesis (see balance of NADHm due to glucose or glutamine in Figure S8a,b for instance). Such a study makes it possible to identify the metabolic pathways at work for the synthesis of a given metabolite. Whenever possible, they are represented in our figures with different colors (green for glutamine and blue for glucose).

With C2M2N, we demonstrated that glutamine is a precursor as good as glucose for the syntheses of the main metabolites necessary for cell proliferation and energy production and we were able to give the quantitative yield in these productions (Table 1). The same yields were obtained with MitoCore that constitutes a cross validation of both models. Taking into account cofactors made it possible to emphasize the role of the malate-aspartate shuttle (MAS) in glucose metabolism by making it a controlling (limiting) step in the use of glucose for metabolic syntheses and energy production, reorienting, when necessary, glycolysis towards lactate production (either the Crabtree effect, Warburg effect, or both). This was also well exemplified by the "gas pedal" mechanism where $\mathrm{Ca}^{2+}$ activates the glutamate/aspartate carrier (T4) enhancing pyruvate formation and mitochondrial respiration [56]. This effect does not occur with glutamine, which can feed TCA cycle without NADHc production. More generally, our study emphasizes the role of mitochondrial transporters which are often antiporters leading to metabolites cycling (colored in gray in the figures) to output other metabolites. One can mention, for instance, glutamate cycling to remove mitochondrial aspartate or malate cycling to remove mitochondrial citrate (e.g., see Figure 6a,b, Figure S6a,b), and also the well-known malate-aspartate shuttle. These cycles could be controlling steps in metabolic network and thus good targets for therapeutic drugs. 
C2M2N takes into account the constraints introduced by the nitrogen metabolism which appear in the simultaneous presence of glucose and glutamine for biomass synthesis (Section 3.8). We quantitatively evidenced the role of ammonia in the microenvironment and its possible recycling inside the same cell or between cells of the same tumor as recently demonstrated [47]. We also showed the role of glutamine in complementing arginine deprivation as evidenced in [54], for instance.

In line with [55], we used C2M2N as a quantitative model to study the impact of a given amount of respiratory chain complexes on the relative uptake of glucose and glutamine and the release of lactate known as the Warburg effect. The Warburg effect clearly appears in our model as a way to resolve the tensions arising from an increased demand in ATP and the necessary reoxidation of the reducing power of proliferating cells. C2M2N allowed us to depict the causal link leading to the Warburg effect, i.e., when the activity of respiratory chain cannot be increased upon an increase in biomass production rate, the oxidative phosphorylation limits both the NADHm reoxidation and the percentage of mitochondrial ATP production. The only way to supply the extra demand in ATP is through glycolysis with an increased uptake of glucose but also an increased production of NADHc, which cannot be reoxidized by the respiratory chain. NADHc is thus reoxidized due to LDH with the release of lactate that dissipates the extra glucose carbons. Others sources of carbons, among which glutamine uptake, are thus necessary to sustain an increase in biomass production rate. In parallel, glutamine acts as an NH3 group donor but can also modulate the cell redox state through a balance between its oxidative or reducing catabolism. Of importance is the possible trend of glutamine to answer the increase in reducing power in making NADPH mainly through IDH1 or ME1; NADPH can be then reoxidized in fatty acids and glutathione syntheses.

C2M2N allowed us to look for the steps whose inhibition could decrease biomass production and, thus, cell proliferation. In our model, the inhibition of any pathway leading to a biomass component will inhibit cell proliferation. This is a good indication of the main targets for anticancer drugs which has to be nevertheless considered with caution because several other minor pathways may exist which are not considered in C2M2N but can be overexpressed in pathological cells. However, it appears in our model that the inhibition of the first steps of glutaminolysis, GLS1 and Glud1 (see Section 3.8), are not sufficient to inhibit biomass formation. Several steps of inhibitions are necessary for this aim. This point would be more evident on genome-scale models showing that C2M2N utilization does not exempt from the consideration of larger models. Nevertheless, C2M2N can constitute a first step to analyze experimental results with the quantitative constraints restricting the study to the possible relevant solutions. It can also be a first step in analyzing the solutions of genome-scale metabolic models by the recognition of common solutions and the reasons of non-common ones. Passing through a midsize metabolic model, such as MitoCore, can be an additional option with an increase by an order of magnitude in the number of reactions (77 reactions for C2M2N, 407 for MitoCore, and around 7440 for Recon 2 [4]).

A drawback of genome-scale models, and of large models in general (more than several hundreds of reactions), is that flux balance analysis is the only possible approach. The number of elementary flux modes (EFMs) is usually out of calculation, and taking into account the rate equations in differential equations, reflecting the change in the concentration of metabolites, faces a number of difficulties (their number, the knowledge of all kinetic constants, etc.). Similarly, metabolic control analysis (MCA) which can give an idea of pertinent therapeutic targets is unrealizable. These tasks are possible and easy with smaller metabolic models restricted to well-known reactions. The interest of having different approaches to explore a metabolic network is to be able to choose the most appropriate method for each particular biological question and, more generally, to confront the solutions given by the different theoretical approaches.

We think that $\mathrm{C} 2 \mathrm{M} 2 \mathrm{~N}$ is a good compromise between a comprehensive compilation of all the metabolic steps and a much lower number of the most important reactions of central carbon and nitrogen metabolism that can give a realistic and quantitative representation of metabolism in many physiological or pathological conditions. 
Supplementary Materials: The following are available online at http://www.mdpi.com/2218-1989/9/5/81/s1, Supplementary Materials 1: Others solutions for pyruvate synthesis from glutamine, Supplementary Materials 2: Decomposition of the metabolic network of Figure $8 \mathrm{c}$ in elementary flux modes, Figure S1: The C2M2N metabolic network, Figure S2: The complete C2M2N metabolic network of energy production from glutamine and glucose, Figure S3: The complete C2M2N metabolic network of pyruvate synthesis from glutamine and glucose, Figure S4: The complete C2M2N metabolic network of aspartate synthesis from glutamine and glucose, Figure S5: The complete C2M2N metabolic network of nucleotides synthesis from glutamine and glucose, Figure S6: The complete C2M2N metabolic network of Fatty Acids synthesis from glutamine and glucose, Figure S7: The complete C2M2N metabolic network of serine synthesis from glutamine and glucose, Figure S8: The complete C2M2N metabolic network of biomass production $(\mathrm{BM}=1)$ with various amount of glutamine and glucose together, Figure S9: The complete C2M2N metabolic network of high biomass production $(\mathrm{BM}=3)$ with various amount of glutamine and glucose together.

Author Contributions: Conceptualization, methodology and writing original draft: J.P.M., writing-review and editing: SR, validation, formal analysis, investigation: JPM and S.R.

Funding: This work was supported by the Plan cancer 2014-2019 No BIO 201406 and the French Association against Myopathies.

Acknowledgments: We would like to thank several colleagues for helpful discussions on our model: Bertrand Beauvoit, Sophie Colombié, Anne Devin, Martine Dieuaide-Noubhani, Yves Gibon, Christine Nazaret, Pierre Petriacq, Sylvain Prigent, and Michel Rigoulet. Thanks to Anne Devin and Louise Injarabian for English corrections.

Conflicts of Interest: The authors declare no conflict of interest.

\section{Abbreviations}

ACS

AGC

AKG

ANT

ARGASE

ASS1_ASL_FH

ASYNT

ASPUP

ATPASE

BM

CL (ACL)

CPS1_OTC

CS

DPH

DPSI

ENOMUT

ETC

FBA

FVA

G1

G2

G3

GG3

GG4

GLS1

GLNUP

GLUCUP

GLUD1
Acetyl-CoA Synthase.

aspartate-glutamate carrier (see T4 in Appendix A)

$\alpha$-ketoglutarate or 2-oxoglutarate

ADP/ATP exchanger.

arginase

argininosuccinate synthase + argininosuccinate lyase + fumarate

hydratase

ATP Synthase.

Uptake of aspartate.

ATP usage.

0.18 SERc + 0.11 Palmitate_c + 0.04 XTPc + 0.19 GLNc + 0.17

$\mathrm{GLUTc}+0.7 \mathrm{ASPc}+0.05 \mathrm{ARGc}+0.12 \mathrm{PYRc}+4 \mathrm{ATPc}=>1$

Biomass $+4 \mathrm{ADPc}+4$ Pic.

(ATP) Citrate Lyase.

carbamoyl phosphate synthase $1+$ Ornithine transcarbamylase

Citrate Synthase.

$\mathrm{pH}$ difference between inside and outside mitochondria.

mitochondrial membrane potential.

Enolase + Phosphoglycerate Mutase.

Electron Transport Chain or Respiratory Chain

Flux Balance Analysis.

Flux Variability Analysis.

hexokinase + phosphoglucose isomerase.

phosphofructokinase + aldolase + triose-phosphate isomerase.

Glyceraldehyde-3P Dehydrogenase + phosphoglycerate kinase.

triose phosphate isomerase + aldolase + fructose

-1,6-biphosphatase.

phosphogluco isomerase + glucose-6-phosphatase.

Glutaminase.

Uptake of Glutamine.

Uptake of glucose.

Glutamate Dehydrogenase. 


\begin{tabular}{|c|c|}
\hline GOT & Glutamate Oxaloacetate Transaminase. \\
\hline IDH & Aconitase + Isocitrate dehydrogenase. \\
\hline $\mathbf{L}$ & Proton leak of the mitochondrial membrane. \\
\hline LACIO & Input/Output of lactate. \\
\hline LDH & Lactate Dehydrogenase. \\
\hline MAS & Malate/Aspartate Shuttle. \\
\hline ME & Malic Enzyme. \\
\hline MDH & Malate Dehydrogenase \\
\hline NAGS_ACY & $\begin{array}{l}\mathrm{N} \text {-acetylglutamate synthase + Amino Acylase (ornithine } \\
\text { synthesis from glutamate). }\end{array}$ \\
\hline NIG & for nigericine, exchange of DPH and DPSI. \\
\hline NOS & NO synthase. \\
\hline NUC & Nucleotide (XTP) Synthesis. \\
\hline OGC & Oxoglutarate carrier (see T2 in Appendix A). \\
\hline ORNT1 & Ornithine/Citrulline $+\mathrm{H}+$ exchanger. \\
\hline ORNT2 & Ornithine/H+ exchanger. \\
\hline PDH & Pyruvate Dehydrogenase. \\
\hline PEPCK & PhosphoEnolPyruvate Carboxy Kinase. \\
\hline PK & Pyruvate Kinase. \\
\hline PL1 & Synthesis of PhosphoLipids. \\
\hline PP1 & Oxidative part of PPP. \\
\hline PP2 & non-oxidative part of PPP. \\
\hline PPP & Pentose Phosphate Pathway. \\
\hline PYC & Pyruvate Carboxylase. \\
\hline RC1 & Complex I of Respiratory Chain. \\
\hline $\mathrm{RC2}$ & succinate dehydrogenase + fumarase. \\
\hline RC34 & Complex III+IV of Respiratory Chain. \\
\hline SEROUT & Output of serine. \\
\hline SERSYNT & $\begin{array}{l}\text { Serine Synthesis = Dehydrogenase }+ \text { Transaminase and } \\
\text { Phosphatase. }\end{array}$ \\
\hline SLP (Substrate Level Phosphorylation) & 2-oxoglutarate dehydrogenase + succinate thiokinase \\
\hline XTP & Nucleotides \\
\hline
\end{tabular}

\section{Appendix A. METATOOL ENTRY FILE OF C2M2N}

-ENZREV

ANT ASS1_ASL_FH ASYNT ENOMUT G3 GLUD1 GOT1 GOT2 IDH1 IDH2 IDH3 LACIO LDH MDH1

MDH2 ME1 ME2 NIG NNT ORNT1 ORNT2 PP2 RC1 RC2 T1 T2 T3 T5 T7 T8 T9 T12 T14 T15 T16 T17 T20 -ENZIRREV

ACS ARGASE ASPUP ATPASE BM CL CPS1_OTC CS G1 G2 GG3 GG4 GLNUP GLS1 GLUCUP GS1 L NAGS ACY NOS NUC PDH PEPCK1 PEPCK2 PK PL1 PL2 PL3 PP1 PYC RC34 SEROUT SERSYNT SLP T4 T6

T11 T13 T18 T19

-METINT

3PG ACETm ACoAc ACoAm ADPc ADPm AKGc AKGm ARGc ASPc ASPm ATPc ATPm CITc CITm CITRc CITRm DPH DPSI G3P G6P GLNc GLNm GLUCc GLUTc GLUTm LACc MALc MALm NADc NADHc NADm NADHm NADPc NADPm NADPHc NADPHm OAAc OAAm ORNc ORNm NH3 Palmitate_c PEPc PEPm Pic Pim PYRc PYRm R5P SERc SUCCm UREAc XTPc

-METEXT

ARG ASP Biomass CO2 CoAc CoAm GLN GLUC GLUT HCO3 LAC NH3_out NO O PalCoAc PalCoAm Palmitate Pi PYR Q QH2 SER UREA XTP -CAT

ACS: ACETm + 2 ATPm + CoAm $=$ ACoAm + 2 ADPm +2 Pim

ANT: ATPm + ADPc + DPSI $=$ ATPc + ADPm

ARGASE: $\mathrm{ARG} c=\mathrm{UREAc}+\mathrm{ORNc}$

ASPUP: ASP = ASPC

ASS1_ASL_FH: CITRc + ASPc +2 ATPc $=$ ARGc + MALc +2 ADPc +2 Pic

ASYNT: 3 ADPm +3 Pim +8 DPH +8 DPSI $=3$ ATPm

ATPASE: ATPc $=$ ADPc + Pic

BM: 0.18 SERc + 0.11 Palmitate_c + 0.04 XTPc + 0.19 GLNc + 0.17 GLUTc + 0.7 ASPc + 0.05 ARGc + 0.12

$\mathrm{PYRc}+4 \mathrm{ATPc}=1$ Biomass $+4 \mathrm{ADPc}+4 \mathrm{Pic}$ 
$\mathrm{CL}: \mathrm{CITc}+\mathrm{ATPc}+\mathrm{CoAc}=\mathrm{ACoAc}+\mathrm{OAAc}+\mathrm{ADPc}+\mathrm{Pic}$

CPS1_OTC: $\mathrm{NH} 3+\mathrm{HCO} 3+$ ORNm +2 ATPm $=$ CITRm +2 ADPm +2 Pim

CS: $\mathrm{ACOAm}+\mathrm{OAAm}=\mathrm{CITm}$

ENOMUT: $P E P C=3 P G$

G1: GLUCc + ATPc $=$ G6P + ADPc

G2: G6P + ATPC $=2$ G3P + ADPc

G3: G3P + NADc + ADPc + Pic $=3 P G+N A D H c+A T P c$

GG3: 2 G3P = G6P + Pic

GG4: G6P $=$ GLUCc + Pic

GLNUP: GLN = GLNc

GLS1: GLNm = GLUTm + NH3

GLUCUP: GLUC = GLUCc

GLUD1: GLUTm + NADm $=$ AKGm + NADHm + NH3

GOT1: GLUTc + OAAc $=$ ASPc + AKGc

GOT2: GLUTm + OAAm = ASPm + AKGm

GS1: GLUTC + NH3 + ATPc $=$ GLNc + ADPc + Pic

IDH1: $\mathrm{CITC}+\mathrm{NADPc}=\mathrm{AKGc}+\mathrm{NADPHc}+\mathrm{CO} 2$

IDH2: $\mathrm{CITm}+\mathrm{NADPm}=\mathrm{AKGm}+\mathrm{NADPHm}+\mathrm{CO} 2$

IDH3: $\mathrm{CITm}+\mathrm{NADm}=\mathrm{AKGm}+\mathrm{NADHm}+\mathrm{CO} 2$

$\mathrm{L}: \mathrm{DPSI}+\mathrm{DPH}=$

LACIO: $\mathrm{LACC}=\mathrm{LAC}$

LDH: PYRc + NADHc $=$ LACc + NADc

MDH1: MALc + NADc $=$ OAAc + NADHc

MDH2: MALm + NADm = OAAm + NADHm

ME1: MALc + NADPc $=$ PYRc + NADPHc + CO2

ME2: MALm + NADm $=$ PYRm + NADHm + CO2

NAGS_ACY: 2 GLUTm + ACoAm + NADHm + 3 ATPm $=$ ORNm + CoAm + NADm + 3 ADPm + 3 Pim + $\mathrm{AKGm}+\mathrm{ACETm}$

NIG: DPSI $=4 \mathrm{DPH}$

NNT: NADHm + NADPm + DPH + DPSI $=$ NADm + NADPHm

NOS: $\mathrm{ARG}+1.5 \mathrm{NADPHc}+4 \mathrm{O}=\mathrm{CITRc}+1.5 \mathrm{NADPc}+\mathrm{NO}$

NUC: R5P + 2 GLNc + ASPc + 6 ATPc + 0.2 NADc + 0.2 Q = XTPc + 2 GLUTc + 6 ADPc + 6 Pic + $0.2 \mathrm{NADHc}$ $+0.2 \mathrm{QH} 2$

ORNT1: ORNc + CITRm $=$ ORNm + CITRc + DPH

ORNT2: ORNc $=$ ORNm + DPH

PDH: PYRm + NADm $=$ ACoAm + NADHm + CO2

PEPCK1: OAAc + ATPc $=$ PEPc + ADPc + CO2

PEPCK2: OAAm + ATPm $=$ PEPm + ADPm + CO2

PK: PEPc + ADPc $=$ PYRc + ATPc

PL1: 8 ACoAc + 7 ATPc + 14 NADPHc + 7 HCO3 = Palmitate $\_$c +7 ADPc + 7 Pic + 14 NADPc + 8 CoAc + $7 \mathrm{CO} 2$

PL2: PalCoAm + 7 NADm + 7 CoAm + $7 \mathrm{Q}=7$ NADHm + 8 ACoAm + 7 QH2

PL3: Palmitate $\_$c + CoAc $=$PalCoAc

PP1: G6P + 2 NADPc $=$ R5P + 2 NADPHc + CO2

PP2: 3 R5P = 2 G6P + G3P

PYC: PYRm + HCO3 + ATPm = OAAm + Pim + ADPm

$\mathrm{RC} 1: \mathrm{NADHm}+\mathrm{Q}=\mathrm{NADm}+\mathrm{QH} 2+4 \mathrm{DPH}+4 \mathrm{DPSI}$

$\mathrm{RC} 2: \mathrm{SUCCm}+\mathrm{Q}=\mathrm{MALm}+\mathrm{QH} 2$

RC34: $\mathrm{QH} 2+\mathrm{O}=\mathrm{Q}+6 \mathrm{DPH}+6 \mathrm{DPSI}$

SEROUT: $S E R C=$ SER

SERSYNT: $3 P G+$ GLUTc + NADc $=$ SERc + AKGc + NADHc + Pic

SLP: $A K G m+\mathrm{NADm}+\mathrm{Pim}+\mathrm{ADPm}=\mathrm{SUCC} \mathrm{m}+\mathrm{NADHm}+\mathrm{CO} 2+\mathrm{ATPm}$

T1: $\mathrm{CITm}+\mathrm{MALc}=\mathrm{CITc}+\mathrm{MALm}+\mathrm{DPH}$

T2: $\mathrm{AKGc}+\mathrm{MALm}=\mathrm{AKGm}+\mathrm{MALc}$

T3: MALm + Pic $=$ MALc + Pim

T4: GLUTc + ASPm + DPH + DPSI $=$ GLUTm + ASPc

T5: Pic + DPH = Pim

T6: $P Y R c+D P H=P Y R m$

T7: PEPm + CITc + DPH + DPSI $=$ PEPc + CITm

T8: GLNC $=$ GLNm

T9: GLUTC + DPH = GLUTm

T11: $\mathrm{PalCoAc}=\mathrm{PalCoAm}$

T12: $\mathrm{Pi}=\mathrm{Pic}$

T13: $\mathrm{XTPC}=\mathrm{XTP}$

T14: $\mathrm{ASPc}=\mathrm{ASP}$

T15: GLUTC = GLUT

T16: $P Y R c=P Y R$ 
T17: Palmitate $c=$ Palmitate

T18: $\mathrm{ARGC}=\mathrm{ARG}$

T19: UREAc $=$ UREA

T20: NH3_out $=$ NH3

\section{References}

1. Orth, J.D.; Thiele, I.; Palsson, B.Ø. What is flux balance analysis? Nat. Biotechnol. 2010, 28, 245-248. [CrossRef]

2. Swainston, N.; Smallbone, K.; Hefzi, H.; Dobson, P.D.; Brewer, J.; Hanscho, M.; Zielinski, D.C.; Ang, K.S.; Gardiner, N.J.; Gutierrez, J.M.; et al. Recon 2.2: From reconstruction to model of human metabolism. Metab. Off. J. Metab. Soc. 2016, 12, 109. [CrossRef] [PubMed]

3. Duarte, N.C.; Becker, S.A.; Jamshidi, N.; Thiele, I.; Mo, M.L.; Vo, T.D.; Srivas, R.; Palsson, B.Ø. Global reconstruction of the human metabolic network based on genomic and bibliomic data. Proc. Natl. Acad. Sci. USA 2007, 104, 1777-1782. [CrossRef]

4. Thiele, I.; Swainston, N.; Fleming, R.M.T.; Hoppe, A.; Sahoo, S.; Aurich, M.K.; Haraldsdottir, H.; Mo, M.L.; Rolfsson, O; Stobbe, M.D.; et al. A community-driven global reconstruction of human metabolism. Nat. Biotechnol. 2013, 31, 419-425. [CrossRef] [PubMed]

5. Ma, H.; Sorokin, A.; Mazein, A.; Selkov, A.; Selkov, E.; Demin, O.; Goryanin, I. The Edinburgh human metabolic network reconstruction and its functional analysis. Mol. Syst. Biol. 2007, 3, 135. [CrossRef]

6. Mardinoglu, A.; Agren, R.; Kampf, C.; Asplund, A.; Nookaew, I.; Jacobson, P.; Walley, A.J.; Froguel, P.; Carlsson, L.M.; Uhlen, M.; et al. Integration of clinical data with a genome-scale metabolic model of the human adipocyte. Mol. Syst. Biol. 2013, 9, 649. [CrossRef] [PubMed]

7. Mardinoglu, A.; Agren, R.; Kampf, C.; Asplund, A.; Uhlen, M.; Nielsen, J. Genome-scale metabolic modelling of hepatocytes reveals serine deficiency in patients with non-alcoholic fatty liver disease. Nat. Commun. 2014, 5, 3083. [CrossRef] [PubMed]

8. Uhlén, M.; Fagerberg, L.; Hallström, B.M.; Lindskog, C.; Oksvold, P.; Mardinoglu, A.; Sivertsson, Å.; Kampf, C.; Sjöstedt, E.; Asplund, A.; et al. Proteomics. Tissue-based map of the human proteome. Science 2015, 347, 1260419. [CrossRef]

9. Yizhak, K.; Chaneton, B.; Gottlieb, E.; Ruppin, E. Modeling cancer metabolism on a genome scale. Mol. Syst. Biol. 2015, 11, 817. [CrossRef]

10. Bordbar, A.; Monk, J.M.; King, Z.A.; Palsson, B.O. Constraint-based models predict metabolic and associated cellular functions. Nat. Rev. Genet. 2014, 15, 107-120. [CrossRef] [PubMed]

11. Smith, A.C.; Eyassu, F.; Mazat, J.P.; Robinson, A.J. MitoCore: A curated constraint-based model for simulating human central metabolism. BMC Syst. Biol. 2017, 11, 114. [CrossRef] [PubMed]

12. Mitchell, P. Coupling of phosphorylation to electron and hydrogen transfer by a chemi-osmotic type of mechanism. Nature 1961, 191, 144-148. [CrossRef]

13. Schuster, S.; Dandekar, T.; Fell, D.A. Detection of elementary flux modes in biochemical networks: A promising tool for pathway analysis and metabolic engineering. Trends Biotechnol. 1999, 17, 53-60. [CrossRef]

14. Metallo, C.M.; Gameiro, P.A.; Bell, E.L.; Mattaini, K.R.; Yang, J.; Hiller, K.; Jewell, C.M.; Johnson, Z.R.; Irvine, D.J.; Guarente, L.; et al. Reductive glutamine metabolism by IDH1 mediates lipogenesis under hypoxia. Nature 2011, 481, 380-384. [CrossRef] [PubMed]

15. Mullen, A.R.; Wheaton, W.W.; Jin, E.S.; Chen, P.H.; Sullivan, L.B.; Cheng, T.; Yang, Y.; Linehan, W.M.; Chandel, N.S.; DeBerardinis, R.J. Reductive carboxylation supports growth in tumour cells with defective mitochondria. Nature 2011, 481, 385-388. [CrossRef] [PubMed]

16. Cluntun, A.A.; Lukey, M.J.; Cerione, R.A.; Locasale, J.W. Glutamine Metabolism in Cancer: Understanding the Heterogeneity. Trends Cancer 2017, 3, 169-180. [CrossRef]

17. Eagle, H. The specific amino acid requirements of a human carcinoma cell (Stain HeLa) in tissue culture. J. Exp. Med. 1955, 102, 37-48. [CrossRef]

18. Kvamme, E.; Svenneby, G. Effect of anaerobiosis and addition of keto acids on glutamine utilization by Ehrlich ascites-tumor cells. Biochim. Biophys. Acta 1960, 42, 187-188. [CrossRef]

19. Daye, D.; Wellen, K.E. Metabolic reprogramming in cancer: Unraveling the role of glutamine in tumorigenesis. Semin. Cell Dev. Biol. 2012, 23, 362-369. [CrossRef]

20. Altman, B.J.; Stine, Z.E.; Dang, C.V. From Krebs to clinic: Glutamine metabolism to cancer therapy. Nat. Rev. Cancer 2016, 16, 773. [CrossRef] 
21. Fiermonte, G.; Palmieri, L.; Todisco, S.; Agrimi, G.; Palmieri, F.; Walker, J.E. Identification of the mitochondrial glutamate transporter. Bacterial expression, reconstitution, functional characterization, and tissue distribution of two human isoforms. J. Biol. Chem. 2002, 277, 19289-19294. [CrossRef]

22. Chen, Q.; Kirk, K.; Shurubor, Y.I.; Zhao, D.; Arreguin, A.J.; Shahi, I.; Valsecchi, F.; Primiano, G.; Calder, E.L.; Carelli, V.; et al. Rewiring of Glutamine Metabolism Is a Bioenergetic Adaptation of Human Cells with Mitochondrial DNA Mutations. Cell Metab. 2018, 27, 1007-1025. [CrossRef]

23. Boele, J.; Olivier, B.G.; Teusink, B. FAME, the Flux Analysis and Modeling Environment. BMC Syst. Biol. 2012, 6, 8. [CrossRef] [PubMed]

24. Folger, O.; Jerby, L.; Frezza, C.; Gottlieb, E.; Ruppin, E.; Shlomi, T. Predicting selective drug targets in cancer through metabolic networks. Mol. Syst. Biol. 2011, 7, 501. [CrossRef] [PubMed]

25. Yang, C.; Ko, B.; Hensley, C.T.; Jiang, L.; Wasti, A.T.; Kim, J.; Sudderth, J.; Calvaruso, M.A.; Lumata, L.; Mitsche, M.; et al. Glutamine oxidation maintains the TCA cycle and cell survival during impaired mitochondrial pyruvate transport. Mol. Cell 2014, 56, 414-424. [CrossRef]

26. Fan, J.; Kamphorst, J.J.; Mathew, R.; Chung, M.K.; White, E.; Shlomi, T.; Rabinowitz, J.D. Glutamine-driven oxidative phosphorylation is a major ATP source in transformed mammalian cells in both normoxia and hypoxia. Mol. Syst. Biol. 2013, 9, 712. [CrossRef]

27. Birsoy, K.; Wang, T.; Chen, W.W.; Freinkman, E.; Abu-Remaileh, M.; Sabatini, D.M. An Essential Role of the Mitochondrial Electron Transport Chain in Cell Proliferation Is to Enable Aspartate Synthesis. Cell 2015, 162, 540-551. [CrossRef] [PubMed]

28. Van Vranken, J.G.; Rutter, J. You Down With ETC? Yeah, You Know D! Cell 2015, 162, 471-473. [CrossRef] [PubMed]

29. Sullivan, L.B.; Gui, D.Y.; Hosios, A.M.; Bush, L.N.; Freinkman, E.; Vander Heiden, M.G. Supporting Aspartate Biosynthesis Is an Essential Function of Respiration in Proliferating Cells. Cell 2015, 162, 552-563. [CrossRef]

30. Garcia-Bermudez, J.; Baudrier, L.; La, K.; Zhu, X.G.; Fidelin, J.; Sviderskiy, V.O.; Papagiannakopoulos, T.; Molina, H.; Snuderl, M.; Lewis, C.A.; et al. Aspartate is a limiting metabolite for cancer cell proliferation under hypoxia and in tumours. Nat. Cell Biol. 2018, 20, 775. [CrossRef]

31. Sullivan, L.B.; Luengo, A.; Danai, L.V.; Bush, L.N.; Diehl, F.F.; Hosios, A.M.; Lau, A.N.; Elmiligy, S.; Malstrom, S.; Lewis, C.A.; et al. Aspartate is an endogenous metabolic limitation for tumour growth. Nat. Cell Biol. 2018, 20, 782. [CrossRef] [PubMed]

32. DeBerardinis, R.J.; Mancuso, A.; Daikhin, E.; Nissim, I.; Yudkoff, M.; Wehrli, S.; Thompson, C.B. Beyond aerobic glycolysis: Transformed cells can engage in glutamine metabolism that exceeds the requirement for protein and nucleotide synthesis. Proc. Natl. Acad. Sci. USA 2007, 104, 19345-19350. [CrossRef] [PubMed]

33. Wise, D.R.; Ward, P.S.; Shay, J.E.S.; Cross, J.R.; Gruber, J.J.; Sachdeva, U.M.; Platt, J.M.; DeMatteo, R.G.; Simon, M.C.; Thompson, C.B. Hypoxia promotes isocitrate dehydrogenase-dependent carboxylation of $\alpha$-ketoglutarate to citrate to support cell growth and viability. Proc. Natl. Acad. Sci. USA 2011, 108, 19611-19616. [CrossRef]

34. Gaude, E.; Schmidt, C.; Gammage, P.A.; Dugourd, A.; Blacker, T.; Chew, S.P.; Saez-Rodriguez, J.; O’Neill, J.S.; Szabadkai, G.; Minczuk, M.; et al. NADH Shuttling Couples Cytosolic Reductive Carboxylation of Glutamine with Glycolysis in Cells with Mitochondrial Dysfunction. Mol. Cell 2018, 69, 581-593. [CrossRef]

35. Fendt, S.M.; Bell, E.L.; Keibler, M.A.; Olenchock, B.A.; Mayers, J.R.; Wasylenko, T.M.; Vokes, N.I.; Guarente, L.; Vander Heiden, M.G.; Stephanopoulos, G. Reductive glutamine metabolism is a function of the $\alpha$-ketoglutarate to citrate ratio in cells. Nat. Commun. 2013, 4, 2236. [CrossRef] [PubMed]

36. Corbet, C.; Draoui, N.; Polet, F.; Pinto, A.; Drozak, X.; Riant, O.; Feron, O. The SIRT1/HIF2 $\alpha$ axis drives reductive glutamine metabolism under chronic acidosis and alters tumor response to therapy. Cancer Res. 2014, 74, 5507-5519. [CrossRef]

37. Corbet, C.; Feron, O. Metabolic and mind shifts: From glucose to glutamine and acetate addictions in cancer. Curr. Opin. Clin. Nutr. Metab. Care 2015, 18, 346-353. [CrossRef] [PubMed]

38. Hanse, E.A.; Ruan, C.; Kachman, M.; Wang, D.; Lowman, X.H.; Kelekar, A. Cytosolic malate dehydrogenase activity helps support glycolysis in actively proliferating cells and cancer. Oncogene 2017, 36, 3915-3924. [CrossRef] [PubMed]

39. Kalhan, S.C.; Hanson, R.W. Resurgence of serine: An often neglected but indispensable amino Acid. J. Biol. Chem. 2012, 287, 19786-19791. [CrossRef] [PubMed] 
40. Snell, K. Enzymes of serine metabolism in normal, developing and neoplastic rat tissues. Adv. Enzym. Regul. 1984, 22, 325-400. [CrossRef]

41. Possemato, R.; Marks, K.M.; Shaul, Y.D.; Pacold, M.E.; Kim, D.; Birsoy, K.; Sethumadhavan, S.; Woo, H.K.; Jang, H.G.; Jha, A.K.; et al. Functional genomics reveal that the serine synthesis pathway is essential in breast cancer. Nature 2011, 476, 346-350. [CrossRef] [PubMed]

42. Locasale, J.W.; Grassian, A.R.; Melman, T.; Lyssiotis, C.A.; Mattaini, K.R.; Bass, A.J.; Heffron, G.; Metallo, C.M.; Muranen, T.; Sharfi, H.; et al. Phosphoglycerate dehydrogenase diverts glycolytic flux and contributes to oncogenesis. Nat. Genet. 2011, 43, 869-874. [CrossRef] [PubMed]

43. Pollari, S.; Käkönen, S.M.; Edgren, H.; Wolf, M.; Kohonen, P.; Sara, H.; Guise, T.; Nees, M.; Kallioniemi, O. Enhanced serine production by bone metastatic breast cancer cells stimulates osteoclastogenesis. Breast Cancer Res. Treat. 2011, 125, 421-430. [CrossRef]

44. Beauvoit, B.; Colombié, S.; Issa, R.; Mazat, J.P.; Nazaret, C.; Pérès, S. Human-Scale Metabolic Network of Central Carbon Metabolism. Application to serine metabolism from glutamine in Cancer Cells. In Proceedings of the Évry Spring School on Advances in Systems and Synthetic Biology; Amar, P., Képès, F., Norris, V., Eds.; CCSD: Paris, France, 2016; pp. 37-56. Available online: https://assb.lri.fr/en/index.php (accessed on 25 April 2019).

45. Kalhan, S.C.; Uppal, S.O.; Moorman, J.L.; Bennett, C.; Gruca, L.L.; Parimi, P.S.; Dasarathy, S.; Serre, D.; Hanson, R.W. Metabolic and genomic response to dietary isocaloric protein restriction in the rat. J. Biol. Chem. 2011, 286, 5266-5277. [CrossRef] [PubMed]

46. Takeuchi, Y.; Nakayama, Y.; Fukusaki, E.; Irino, Y. Glutamate production from ammonia via glutamate dehydrogenase 2 activity supports cancer cell proliferation under glutamine depletion. Biochem. Biophys. Res. Commun. 2018, 495, 761-767. [CrossRef]

47. Spinelli, J.B.; Yoon, H.; Ringel, A.E.; Jeanfavre, S.; Clish, C.B.; Haigis, M.C. Metabolic recycling of ammonia via glutamate dehydrogenase supports breast cancer biomass. Science 2017, 358, 941-946. [CrossRef] [PubMed]

48. Eng, C.H.; Yu, K.; Lucas, J.; White, E.; Abraham, R.T. Ammonia derived from glutaminolysis is a diffusible regulator of autophagy. Sci. Signal. 2010, 3, ra31. [CrossRef] [PubMed]

49. Dang, C.V. Feeding frenzy for cancer cells. Science 2017, 358, 862-863. [CrossRef]

50. Martinez-Outschoorn, U.E.; Peiris-Pagés, M.; Pestell, R.G.; Sotgia, F.; Lisanti, M.P. Cancer metabolism: A therapeutic perspective. Nat. Rev. Clin. Oncol. 2017, 14, 11-31. [CrossRef]

51. Süer Gökmen, S.; Yörük, Y.; Cakir, E.; Yorulmaz, F.; Gülen, S. Arginase and ornithine, as markers in human non-small cell lung carcinoma. Cancer Biochem. Biophys. 1999, 17, 125-131.

52. Singh, R.; Pervin, S.; Karimi, A.; Cederbaum, S.; Chaudhuri, G. Arginase activity in human breast cancer cell lines: N(omega)-hydroxy-L-arginine selectively inhibits cell proliferation and induces apoptosis in MDA-MB-468 cells. Cancer Res. 2000, 60, 3305-3312.

53. Keshet, R.; Szlosarek, P.; Carracedo, A.; Erez, A. Rewiring urea cycle metabolism in cancer to support anabolism. Nat. Rev. Cancer 2018, 18, 634. [CrossRef] [PubMed]

54. Long, Y.; Tsai, W.B.; Wangpaichitr, M.; Tsukamoto, T.; Savaraj, N.; Feun, L.G.; Kuo, M.T. Arginine deiminase resistance in melanoma cells is associated with metabolic reprogramming, glucose dependence, and glutamine addiction. Mol. Cancer Ther. 2013, 12, 2581-2590. [CrossRef] [PubMed]

55. Dai, Z.; Shestov, A.A.; Lai, L.; Locasale, J.W. A Flux Balance of Glucose Metabolism Clarifies the Requirements of the Warburg Effect. Biophys. J. 2016, 111, 1088-1100. [CrossRef]

56. Gellerich, F.N.; Gizatullina, Z.; Gainutdinov, T.; Muth, K.; Seppet, E.; Orynbayeva, Z.; Vielhaber, S. The control of brain mitochondrial energization by cytosolic calcium: The mitochondrial gas pedal. IUBMB Life 2013, 65, 180-190. [CrossRef] [PubMed]

(C) 2019 by the authors. Licensee MDPI, Basel, Switzerland. This article is an open access article distributed under the terms and conditions of the Creative Commons Attribution (CC BY) license (http://creativecommons.org/licenses/by/4.0/). 